\title{
Cyclin-dependent kinase 21 is a novel regulator of proliferation and meiosis in the male germline of zebrafish
}

\author{
Kaitlyn A Webster ${ }^{1}+$, Katrin Henke ${ }^{2}$, Delaney M Ingalls ${ }^{1}$, Adeeba Nahrin ${ }^{1}$, Matthew P Harris ${ }^{2}$ \\ and Kellee R Siegfried ${ }^{1}$ \\ ${ }^{1}$ Biology Department, University of Massachusetts Boston, Boston, Massachusetts, USA and ${ }^{2}$ Orthopaedic Research \\ Laboratories, Boston Children's Hospital and Department of Genetics, Harvard Medical School, Boston, \\ Massachusetts, USA \\ Correspondence should be addressed to K R Siegfried; Email: kellee.siegfried@umb.edu
}

${ }^{\dagger}$ (K A Webster is now at John B. Little Center for Radiation Sciences, Harvard T.H. Chan School of Public Health, Boston, Massachusetts, USA)

\begin{abstract}
Germ cell differentiation and maintenance relies on complex regulation of mitotic and meiotic progression. Cyclin-dependent kinases (CDKs) and their activating cyclin partners are known to have specialized roles in regulating cell cycle progression across tissues, including germ cells. Very little is known about CDK/cyclin function in zebrafish or the regulation of germ cell maintenance and differentiation. In a forward genetic screen for gonadogenesis defects in zebrafish, a mutation disrupting cdk21 (cyclin-dependent kinase 21) was identified, which caused gonad hypoplasia, reduced fertility and failure of female sex specification. The cdk21 gene is unique to fishes, though the encoded protein is related to the D-cyclin partners Cdk4 and Cdk6, which are known G1 cell cycle regulators. In the testis, cdk21 mutant germ cells exhibited cell cycle defects such as diminished proliferation, prolonged meiosis and delayed sperm differentiation. Furthermore, cdk21 mutants failed to maintain germ cells following breeding. Based on these findings, we propose that $c d k 21$ regulates spermatogonial proliferation, progression through meiosis and germline stem cell activation in the testis. In addition, we investigated $c d k 4$ and $c d k 6$ in zebrafish development and found that each has distinct expression patterns in the gonads. Mutant analysis demonstrated that $c d k 6$ was necessary for viability beyond larval stages. In contrast, cdk4 mutants were viable but were all male with low breeding success and sperm overabundance. Our analysis demonstrated that zebrafish harbor three genes of the $c d k 4 / 6$ family, $c d k 4, c d k 6$ and $c d k 21$, with $c d k 21$ having an essential role in germ cell development in the testis.

Reproduction (2019) 157 383-398
\end{abstract}

\section{Introduction}

Germ cells are dynamic cells that can be quiescent, proliferative, mitotic or meiotic. Continued production of germ cells is maintained through self-renewal of a germline stem cell population, which is present in both sexes of most animals. In zebrafish testes, the spermatogonial stem cell population and the stages of spermatogenesis have been described using thorough cellular characterization; however, little is known about how these processes are genetically regulated (Leal et al. 2009, Nóbrega et al. 2010, Schulz et al. 2010). In mice, it is well established that single spermatogonia $\left(A_{s}\right)$ are self-renewing and give rise to pairs $\left(A_{p r}\right)$, which then proliferate and differentiate into chains of spermatogonia $\left(A_{a l}\right)$ cells connected by cytoplasmic bridges (de Rooij 2001). Spermatogonia then enter meiosis and, after completing both meiotic divisions, terminally differentiate into sperm. Zebrafish exhibit similar developmental progression from $A_{s}$ through differentiation of sperm (Leal et al. 2009, Nóbrega et al. 2010). Thus, spermatogenesis requires complex changes in cell cycle regulation from stem cell activation and self-renewal, mitotic spermatogonial divisions and meiotic spermatocyte divisions.

Germ cell proliferation and differentiation can influence several aspects of gonadal development, including sex differentiation. In domesticated zebrafish, including common laboratory strains, sex determination is attributed to a polygenic mechanism, whereas wild zebrafish have a ZZ/ZW chromosomal sex determination mechanism (Bradley et al. 2011, Anderson et al. 2012, Howe et al. 2013, Wilson et al. 2014, Luzio et al. 2015). However, in both cases, the genes involved are not known. The presence of the germline is essential for commitment to female sexual fate (Slanchev et al. 2005, Siegfried \& Nüsslein-Volhard 2008, Dranow et al. 2013). Loss of germ cells precludes ovary fate in zebrafish, and high quantity of embryonic germ cells is assumed to be an indicator of eventual 
female sex (Siegfried \& Nüsslein-Volhard 2008, Tzung et al. 2015). In zebrafish, the necessity of the germline in female sex differentiation is attributed to meiotic oocytes, which signal to the somatic gonad in support of ovary fate; however, these female sex-supporting signals have not been identified (Rodríguez-Marí et al. 2010, Dranow et al. 2016). As meiotic germ cells are needed in adequate abundance to specify and maintain ovaries, robust germ cell proliferation and timely initiation of meiosis is a prerequisite for female fate determination in zebrafish.

The timing of meiotic initiation and progression is sexually dimorphic in many animals in order to accommodate the unique processes of gametogenesis in each sex (Morelli \& Cohen 2005). For example, prenatal completion of prophase I and diplotene arrest is observed in mammalian ovaries, whereas male meiosis is initiated postnatally and is continuous. Furthermore, resumption of meiosis in oocytes is tightly regulated such that only one or few oocytes resume meiosis in each reproductive cycle. In zebrafish, both sexes will initially form juvenile ovaries with oocytes actively engaged in meiosis I, irrespective of their later testis or ovary differentiation (Hsu et al. 2018). However, once germ cells are committed to oogenic or spermatogenic development, similar sexually dimorphic regulation of meiotic divisions occurs as in mammals (Lubzens et al. 2010, Schulz et al. 2010). Cell cycle regulators are integral to managing mitotic divisions of gonial cells as well as the controlled resumption of meiosis in females, and the progression through meiosis in both sexes. The coordinated regulation of meiotic progression is necessary for faithful chromosome segregation as well as gametogenesis.

Interestingly, cell cycle regulators such as retinoblastoma (RB1), cyclins (CYC) and cyclindependent kinases (CDKs) were found to be essential for several aspects of germ cell development, including stem cell specification and differentiation of the male germline of mice (Hu et al. 2013, Wolgemuth et al. 2013, Yang et al. 2013, Risal et al. 2016). The canonical cell cycle CDKs include CDK1, 2, 4 and 6 (Malumbres 2014). These kinases are activated by CYCA and B (CDK1), E (CDK2) and D (CDK4 and 6). In particular, these pairings of CYCs and CDKs are necessary for progression through the cell cycle from G0 through mitosis, thus ensuring that processes such as cell growth and DNA replication, segregation and repair occur faithfully. This regulation is conducted in part through relief of transcriptional repression by RB1, which binds and restricts $\mathrm{E} 2 \mathrm{~F}$ transcription factors. RB1 is inactivated by sequential phosphorylation: first, a single phosphate is added during G1 by the G1 CDKs, CDK4 or CDK6, and then hyperphosphorylation is achieved by the G1/S kinase CDK2 in late G1 and throughout $S$ phase (Risal et al. 2016). Thus, the $G 1$ phase and transition to $S$ is controlled through modulation of RB1 by CDK activity.
Here, we describe the function of cyclin-dependent kinase 21 (cdk21), a novel putative cell cycle regulator of the male germline in zebrafish. Homology to Cdk4 and Cdk6 suggests that Cdk21 functions as a G1 cell cycle regulator. We found that loss of cdk21 function resulted in male-only development and impaired fertility. Mutant testes displayed defects during spermatogenesis leading to gradual depletion of germ cells in breeding males. We show that $c d k 21$ functions as a driver of spermatogonial mitosis and is required for timely meiotic progression. As $c d k 21$ is in the $c d k 4 / 6$ gene family, we investigated the expression and function of $c d k 4$ and $c d k 6$ in germ cell development. Here, we report that each gene exhibits sexually dimorphic expression in the gonad and that they have partially but not completely redundant functions. These studies provide new insights into the roles of G1 CDKs in gonad development as well as embryogenesis.

\section{Materials and methods}

\section{Zebrafish strains and husbandry}

Zebrafish were maintained by standard conditions. Institutional Animal Care and Use Committee approval was attained prior to carrying out all animal procedures. Strains and mutant alleles used in this study were as follows: AB, Tübingen, WIK, $c d k 21^{t 30421}, c d k 21^{u m b 7}, c d k 4^{u m b 8}, c d k 6^{u m b 9}$. Animals were killed by prolonged exposure to tricaine (MS-222).

\section{Phylogenetic analyses}

Maximum likelihood analyses were used to infer evolutionary history of CDK orthologues. Bootstrap consensus trees (500 replicates) are presented with nodes less than $70 \%$ confidence collapsed. 241 positions were used in final analyses. For unrooted analyses of zebrafish CDKs, neighbor joining method was used with distance to scale of amino acid substitutions per site. 224 positions were used in the analysis. For both analyses, gaps and missing data were eliminated. All analyses were performed using MEGA7 (Kumar et al. 2016).

\section{Mutagenesis screen, exome capture and sequencing}

A mutagenesis screen to identify gonadogenesis mutants was carried out as part of the ZF-MODELS - Zebrafish Models for Human Development and Disease, project (http://www. zfmodels.org/). Briefly, males of the Tübingen strain were treated five times with 3.3 to $3.5 \mathrm{mM} \mathrm{N}$-Ethyl-N-Nitrosourea (ENU). The F3 generation were raised to approximately 3 months post fertilization (mpf) and gonads were viewed for morphological defects by dissection. Those exhibiting drastically reduced size or apparently lacking mature sperm (as determined by a less opaque appearance) were analyzed by histology. The t30421 mutation was isolated based on having small testes with abnormal appearing germ cells.

Genomic DNA from 20F2 orF3 phenotypic mutants stemming from a WIK outcross was extracted from fin tissue using the Qiagen DNeasy Blood and Tissue kit. DNA was pooled with 
an equal amount for each individual. For library preparation $5 \mu \mathrm{g}$ of pooled genomic DNA was sheared to an average size of $200 \mathrm{bp}$ using a Covaris sonicator (Covaris, Woburn, MA, USA). Barcoded sequencing libraries were prepared using the KAPA Hyper Prep Kit (Kapa Biosystems, Wilmington, MA, USA) following the manufacturer's instructions.

For exon capture we used a custom Agilent Technologies $1 \mathrm{M}$ SureSelect DNA capture array (Daane et al. 2016), including all coding regions of the zv9 assembly of the zebrafish genome. The capture was performed using the Agilent Oligo aCGH/ Chip-on-chip Hybridization kit. 50 bp paired end sequencing was performed on an Illumina HiSeq machine (Illumina, San Diego, CA, USA), resulting in 75.6 million reads and an average exome coverage of $18 \times$.

\section{Linkage analysis and mutation identification}

The causative $t 30421$ mutation was mapped from the exome sequence data as described (Bowen et al. 2012). In brief, sequencing reads were aligned to the zebrafish genome, SNPs were identified using GATK and a mapping score was calculated based on homogeneity-by-descent. Nonsynonymous mutations were then identified within regions with high mapping scores. Linkage was confirmed by sequencing identified SNPs in the region in 20 homozygous mutants and 20 phenotypically wild-type siblings. Further analysis of the SNP at the $c d k 21$ locus in additional samples demonstrated that this mutation was tightly linked to the t30421 mutant phenotype.

\section{Fertility assays}

One adult female and male were paired in mating boxes containing plastic grass for enrichment. The following day, all eggs were collected and transferred to a petri dish of E3 buffer in a $28^{\circ} \mathrm{C}$ incubator. At $4-6 \mathrm{~h}$ post fertilization, the number of cleavage through gastrulation stage embryos were counted as successfully fertilized. Eggs appearing cloudy, degenerated and without cleavage were considered unfertilized. Clutch sizes of at least 100 eggs were included in analyses, except where otherwise noted, and percent fertilized represents all successfully fertilized eggs out of the total clutch size.

\section{CRISPR/Cas9 gene editing}

All mutant alleles excluding $c d k 21^{\text {t30421 }}$ were generated using CRISPR/Cas9 genome editing. sgRNA target sites were selected using CRISPRscan (Moreno-Mateos et al. 2015). Alt-R Custom CRISPR-Cas9 crRNAs from Integrated DNA Technologies(Coralville, IO, USA) were ordered for each gene/target site, reconstituted and duplexed with tracrRNA according to manufacturer instructions. Duplexed sgRNAs were co-injected with Transfection-ready Cas9 SmartNuclease mRNA (System Biosciences, Pale Alto, CA, USA, CAS500A-1) into one-cell stage embryos of the $A B$ wild-type strain. Mutations were detected by analysis of PCR products on $6 \%$ TAE acrylamide gels. Guide RNA target sequences were as follows: cdk21 - GACCACATTTCTCAG; cdk4 GTTCTGCGGAGATTC; cdk6 - GCTCGGGATTTGAAG.
All mutant alleles found in genomic DNA were confirmed to cause the predicted changes in the mRNA by sequencing cDNA derived from heterozygous or mutant fish.

\section{Genotyping fish to detect mutations}

All genotyping primers are listed in Supplementary Table 1 (see section on Supplementary data given at the end of this article). To genotype $c d k 21^{t 30421}$ mutants, exon 4 of $c d k 21$ was amplified by PCR and either sequenced to identify homozygosity for the mutation or digested with the restriction enzyme BseYI, which cuts the wild-type but not the $c d k 21^{t 30421}$ allele. To genotype $c d k 21^{u m b 7}$ mutants, exon 2 was PCR amplified and digested with Ddel, which does not cut the mutant allele. The $c d k 4^{u m b 8}$ mutation was identified by PCR amplification of exon 5 and digested with Hpy188I, which does not cut the mutant allele. The $c d k 6^{u m b 9}$ mutation was identified by PCR amplification of exon 1 and digested with Bsll, which cuts two sites in the mutant allele, rather than three as in the wild type.

\section{In situ hybridizations}

In situ hybridizations performed on cryosections were conducted according to Smith et al. (Smith et al. 2008). For ISH on sections, tissue was fixed overnight in $4 \%$ paraformaldehyde (PFA), embedded in OCT and processed for cryosectioning. Sense and antisense probes for vasa, cdk21, cdk4, cdk6 and rb1 were synthesized based on partial cDNAs from each respective gene, amplified using the RT-PCR primers listed in Supplementary Table 1. RT-PCR products were subcloned into pGemT-Easy (Promega), and the Roche DIG RNA Labeling Kit (Roche 11175025910) was used for generating probes according to manufacturer specifications. Detection was carried out using an alkaline phosphatase-conjugated anti-Digoxigenin antibody and visualized with commercial BM Purple. Sense controls were run for all experiments and exhibited no staining (data not shown).

\section{Histological analysis, immunohistochemistry and immunofluorescence}

Fish were killed in tricaine (MS-222) solution prior to dissection of whole trunks. For adults, abdomens were opened and gonads examined to determine sex prior to fixation. All trunks were fixed $5 \mathrm{~h}$ at room temperature in modified Davidson's fixative $(500 \mathrm{~mL} 37 \%$ formalin, $750 \mathrm{~mL} 100 \%$ ethanol, $250 \mathrm{~mL}$ glacial acetic acid, $750 \mathrm{~mL}$ distilled water) and embedded in paraffin prior to sectioning. Sections of $5 \mu \mathrm{m}$ were stained using modified Harris hematoxylin (Sigma HHS16) and Eosin $\mathrm{Y}$, according to standard protocols. Immunohistochemistry was performed on $5 \mu \mathrm{m}$ sections from Davidson's fixed, paraffinembedded zebrafish torsos. Antigen retrieval was done with $\mathrm{pH}$ 6.4 sodium citrate buffer. Primary antibodies against cleaved Caspase-3 (Cell Signaling \#9661) were used at 1:300 dilution with an overnight incubation at $4^{\circ} \mathrm{C}$. Standard detection by horseradish peroxidase and 3,3'-diaminobenzidine was performed following species-specific secondary antibody incubation (Vector Laboratories PK-6100). For immunofluorescence, standard immunohistochemistry was 
performed as described earlier, except for the steps following primary antibody incubation. Primary antibodies for $\alpha$-Tubulin (Sigma T9026) and Synaptonemal complex protein 3 (Sycp3) (Abcam ab150292) were used at concentrations of 1:1000 and 1:200, respectively. Fluorophore-conjugated, speciesspecific secondary antibodies were incubated for $2 \mathrm{~h}$ at room temperature at concentrations recommended by the supplier. Sections were then incubated with DAPI and mounted with Fluoroshield (Sigma-Aldrich F6182). Testes from four wildtype and four cdk21 mutants were imaged and analyzed in IF experiments. Images were captured with a Zeiss AxioObserver Z1 inverted fluorescent microscope equipped with an Apotome 2 structured illumination optical sectioning device, and a Hamamatsu Orca 4 Flash LTE camera (Hamamatsu, Bridgewater, NJ, USA).

\section{BrdU proliferation assay}

Exposure to and incorporation of BrdU was performed by allowing fish to swim in water containing BrdU, according to Leal et al. (2009). Following an 18-h exposure, chase times were either $16 \mathrm{~h}$ or 5 days, to capture spermatogonia and spermatozoa, respectively, as reported in the aforementioned study. Testes were harvested and fixed in Davidson's fixative. Immunohistochemistry was done as described in the immunohistochemistry methods above, using anti-BrdU primary antibody (1:200, Cell Signaling \#5292). Slides stained for BrdU were scanned using Leica Biosystems Aperio ScanScope XT; cells were manually counted using Fiji and percentage of BrdU-positive cells was calculated. A testis from four individuals per condition, one section each, was used for quantification. For quantifying spermatogonia + spermatocytes, the average number of cells counted for each sample was 457 for wild types and 365 for mutants, with a range of 49-658 and 25-365, respectively. For quantifying spermatogonia, the average number of cells counted per sample was 44 for wild types and 57 for mutants, with a range of 22-102 and 21-92, respectively. $P$ values were calculated using the MannWhitney test for unpaired data.

\section{Apoptosis quantification}

Cleaved Caspase-3 labeling was done for 11 and 13 gonads of wild-type and cdk2 $1^{\text {t30421 }}$ mutants, respectively. Counts were within one $40 \times$ field of single 5 micron histological sections of adult testes (one section per fish). The average number of cells counted for each sample was 1933 for wild types and 754 for mutants, with a range of 84-4500 and 54-1587 total cells counted, respectively. Percentage of apoptosis was calculated as the proportion of cleaved Caspase-3-positive cells among total number of cells within the gonad in a given section, $P$ values were calculated using the Mann-Whitney test for unpaired data.

\section{Sperm quantification}

Histological sections were manually annotated to define the area occupied by germ cells (germ cell cysts) and area occupied by sperm. Six individuals were used, one section per individual. The total area tabulated was equal for all sections. The ratio of sperm area to overall area occupied by germ cells (germ cell cysts + sperm) was calculated. $P$ values were calculated using the Mann-Whitney test for unpaired data.

\section{RNA extraction and CDNA synthesis for RT-PCR}

Whole organs were dissected from fish and collected directly into TRI Reagent. A single ovary was used for each RNA extraction while testes were pooled from 3 to 5 males, depending on size. Other tissues were pooled from adult males and females. Embryonic and larval tissues were also pooled from several individuals. Tissues were homogenized with a motorized pestle, and total RNA extraction was performed according to the manufacturer's protocol. First-strand cDNA synthesis was reverse-transcribed from total RNA using AMV Reverse Transcriptase. All RT-PCR experiments were performed on multiple tissue samples. Primers were designed such that amplification from genomic DNA would give a larger band than that expected from cDNA amplification. Primers used for RT-PCR are listed in Supplementary Table 1.

\section{Western blot}

Western blot assaying was performed on protein extracts of pools of whole testes from 3 to 5 males per sample. Testes were homogenized in RIPA buffer (Thermo PI89900) plus Protease Inhibitor (Thermo 88665). Separation of proteins was done on $8 \%$ SDS-polyacrylamide gel by electrophoresis. $\alpha$ Tubulin (Sigma T9026, used as a loading control at 1:1000), Sycp3 (Abcam ab150292, 1:200) and Rb1 (Anaspec, Fremont, CA, USA, 55432, 1:500) were incubated on PVDF membranetransferred blots overnight at $4^{\circ} \mathrm{C}$ and detected using LICOR secondary antibodies and imaging system (LI-COR, Lincoln, NE, USA). Lot numbers for all antibodies were consistent across assays. All Westerns were repeated with at least three biological replicates.

\section{Results}

\section{cdk21 encodes a putative G1 cyclin-dependent kinase and is enriched in spermatogonia}

An ENU mutagenesis screen was conducted with the intent to identify novel regulators of gonad differentiation and germ cell development in zebrafish. A mutant line (t30421) was selected based on the appearance of a dysmorphic and dysgenic testis upon gross dissection. Mutant males had mostly transparent testes of reduced size compared to larger opaque testes of wild-type fish (Fig. 1B" and $\mathrm{C}^{\prime \prime}$ ). Opacity of the testes is conferred by the presence of sperm; thus, mutant males had abnormal germ cell development or did not initiate or maintain spermatogenesis normally, which was supported by histological analysis (Fig. 2). Secondary sex characteristics were normal, including yellow pigmentation and absence of a prominent genital papilla (1A-C'). Notably, abnormal females were never found in the population, which we later found to 

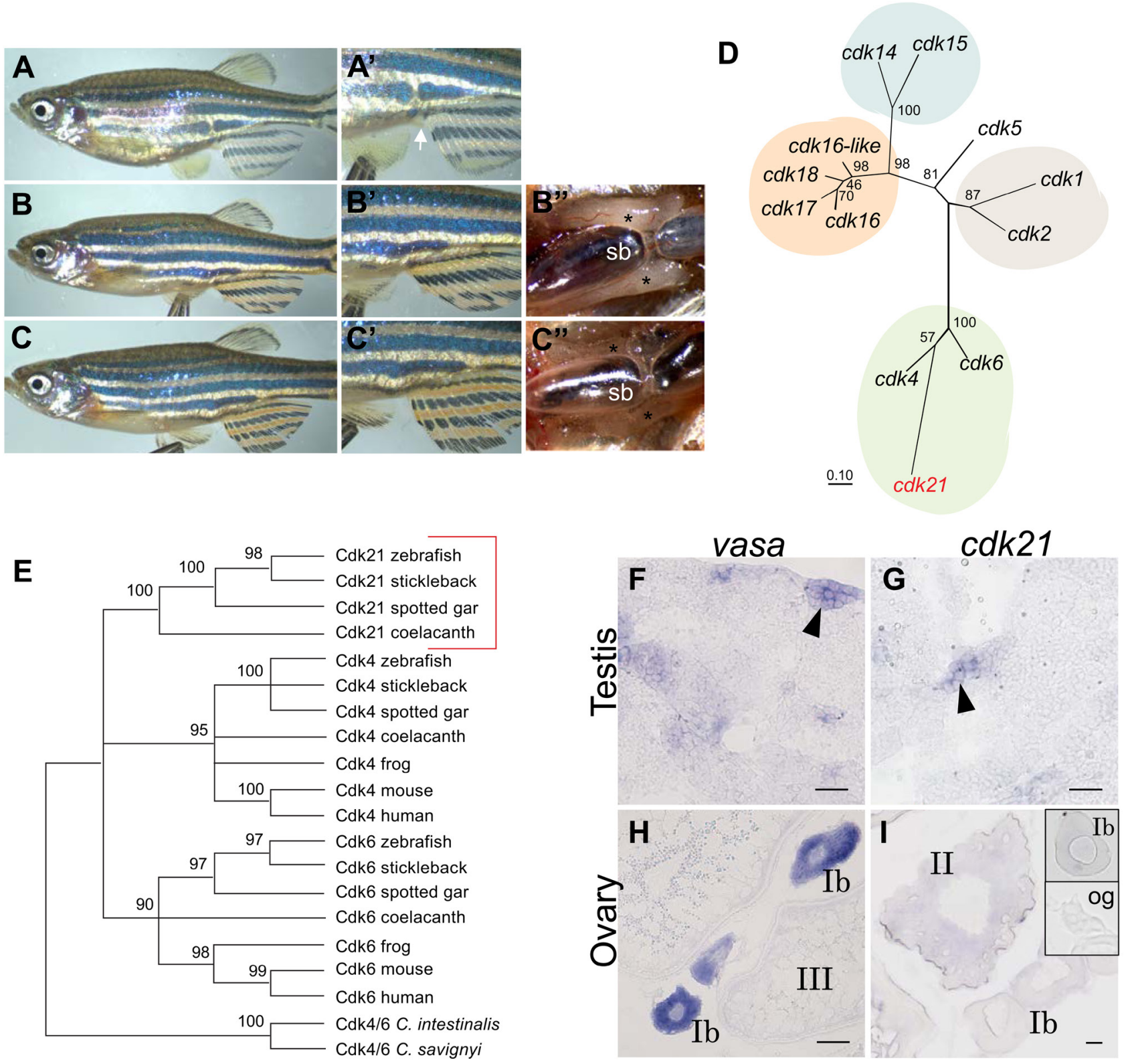

Figure $1 c d k 21$ is a male germ cell enriched G1 cyclin-dependent kinase in fish. (A) Wild-type adult female zebrafish have rounded abdomen (A), little yellow fin pigmentation and an external genital papilla (arrow, $\mathrm{A}^{\prime}$ ). (B) Wild-type adult male zebrafish are slender (B) with prominent yellow pigmentation, no external genital papilla $\left(\mathrm{B}^{\prime}\right)$, and develop paired elongated testes $\left(\mathrm{B}^{\prime \prime}\right)$ which appear opaque when sperm is abundant. (C) Adult cdk21-/- males have male pigmentation $\left(\mathrm{C}^{\prime}\right)$ and clear, hypoplastic testes $\left(\mathrm{C}^{\prime \prime}\right)$. (D) Unrooted maximum likelihood analysis of known zebrafish cell cycle regulating cyclin-dependent kinase proteins shows that Cdk21 clusters with Cdk4 and Cdk6. (E) Cdk21 protein groups with Cdk4 and Cdk6 and was found broadly in bony fishes, but not in tetrapods. The chordate Ciona possess a single Cdk4/6 orthologue. (F, G, H and I) Section in situ hybridization of adult zebrafish gonads. ( $\mathrm{F}$ and $\mathrm{H}$ ) The vasa transcript indicates spermatogonia in the testes (arrowhead, $\mathrm{F}$ ), and stages I and II oocytes in the ovary (H). (G) The cdk21 transcript was expressed in the spermatogonia of the testes (arrowhead, G). (I) In the ovary it was not detected in oogonia (lower inset) but was expressed at low levels in the cytoplasm of stage II oocytes as well as the Balbiani body (upper inset) of stage lb oocytes. Oocyte staging according to Maack \& Segner 2003. *, testes; arrow, genital papilla; sb, swim bladder; arrowhead, spermatogonia; og, oogonia; scale bars = 50 microns.

be due to a failure of $c d k 21$ mutants to specify female sex. Thus, the mutants exhibited defects primarily in testis development.

To identify the causative mutation resulting in gonadal dysgenesis in the $t 30421$ mutant line, an exome sequencing approach was taken (Bowen et al. 2012). A missense mutation disrupting the $c d k 21$ gene was linked to the testicular dysgenesis phenotype, which affected a highly conserved residue in the encoded kinase domain (Supplementary Fig. 1A and C). Due to the replacement of 

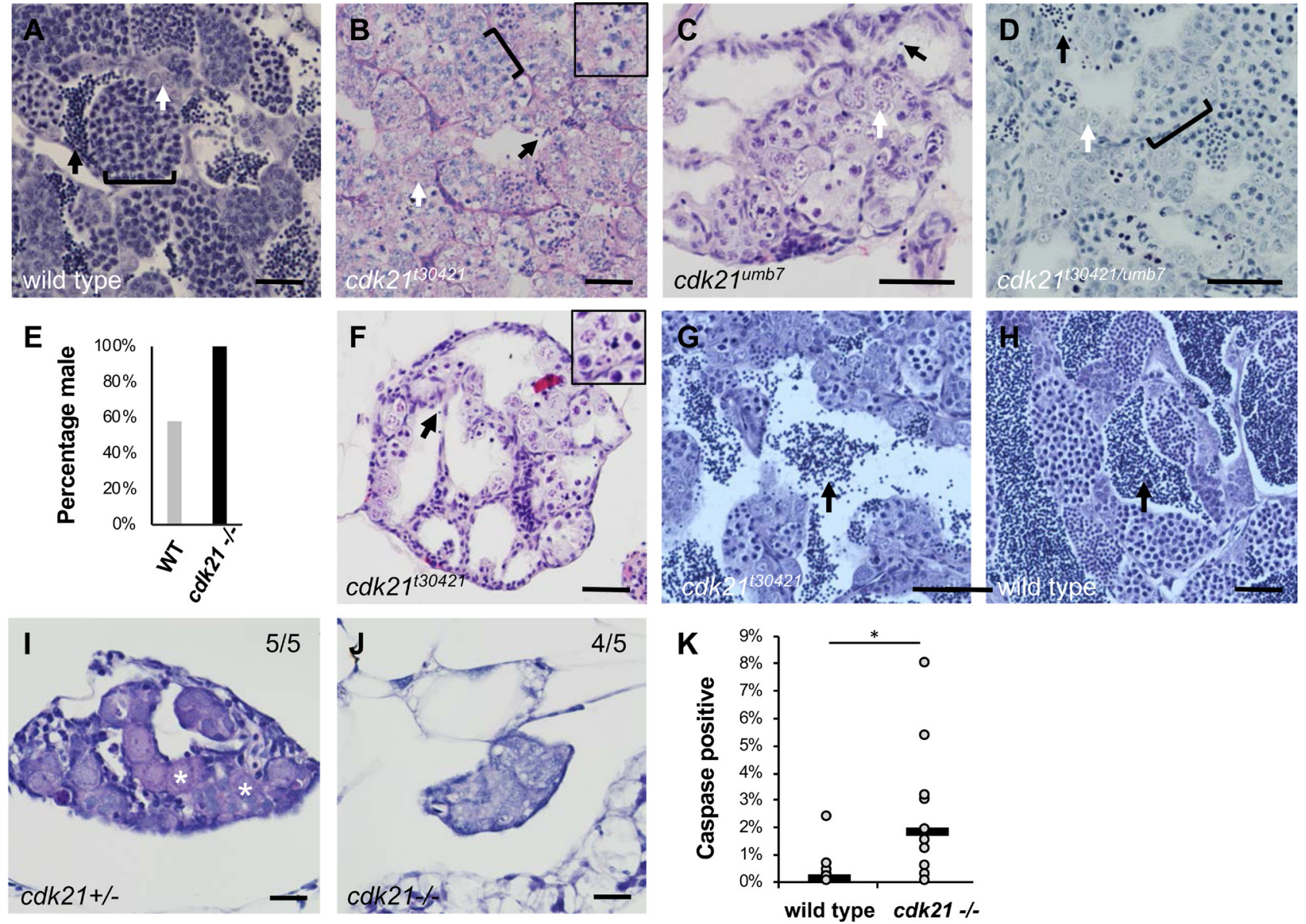

Figure 2 Loss-of-function mutations in the $c d k 21$ gene cause spermatogenesis defects and preclusion of ovary fate. (A, B, C, D and F, G, H) H\&E staining of sections of adult zebrafish testes. (A) Wild-type adult zebrafish testes contained discrete cysts of germ cells ranging from mitotic spermatogonia (white arrow) to meiotic spermatocytes (bracket), and lumens populated by sperm (black arrow). (B, C and D) Adult homozygous $c d k 21^{t 30421}, c d k 21^{u m b 7}$, and transheterozygous $c d k 21^{t 30421 / \mathrm{umb}^{7}}$ testes contained mitotic and meiotic germ cells but displayed little-to-no sperm. Insets show germ cells with abnormal nuclei. (E) $c d k 21^{t 30421}$ mutants were $100 \%$ male. WT includes $c d k 21+/+$ and $c d k 21+/-(N=42)$. (F) $c d k 21^{\text {t30421 }}$ testis following multiple successive breeding were nearly devoid of germ cells. $\left(\mathrm{G}\right.$ and $\mathrm{H}$ ) Testes from one-year-old $c d k 21^{\text {t30421 }}$ mutant (G) and wild-type sibling (H) maintained in the absence of females. (I and J) H\&E stain of juvenile gonads at 3 weeks post fertilization (wpf). (I) 5 of 5 gonads from $3 \mathrm{wpf}$ heterozygous fish contained oocytes and were classified as juvenile ovaries. (J) 4 of $5 \mathrm{cdk} 21^{130421}$ siblings contained only undifferentiated gonial cells within gonads and with no oocytes present. (K) Testes from adult cdk2 $1^{130421}$ had increased amounts of cleaved Caspase-3-positive germ cells; horizontal bars denote the mean $(P=0.017)$. White arrow, spermatogonia; bracket, spermatocytes; black arrow, sperm; asterisk, oocytes; WT, wild-type. Scale bars $=20 \mu \mathrm{m}$.

a small glycine residue with a large aromatic tryptophan residue, we hypothesize that kinase function of Cdk21 is disrupted by this mutation. In order to confirm that the cdk2 $21^{\text {t30421 }}$ mutation caused the male sterility phenotype, a second mutant allele was generated, $C d k 21^{u m b 7}$, using CRISPR/Cas9-mediated gene editing, which resulted in a frame shift mutation at codon 105 leading to a premature truncation within the kinase domain (Supplementary Fig. 1A). Mutants harboring either cdk21 allele were $100 \%$ male $(n>100$ mutant males per genotype) and exhibited similar phenotypic defects (by gross morphology and histological analysis). Furthermore, the mutations failed to genetically complement confirming that these mutations cause the observed defects in the testis (Fig. 2).
CDKs are found across species, and this gene family has widely expanded and includes more than 20 genes with diverse functions. The $c d k 21$ gene had not been previously described in any species. BLAST analysis identified $c d k 21$ in basal ray finned fish (spotted gar) as well as early lobe finned fish (coelacanth) where it is present as a single-copy gene. However, we were unable to identify cdk21 in mammals or other tetrapods. The presence of the cdk21 gene in the coelacanth, which shares a common ancestor with tetrapods, suggests that this gene was ancestral in vertebrates but lost in the tetrapod lineage (Fig. 1E). Interestingly, Cdk21 is homologous to the major cell cycle-regulating Cdks and clusters with the G1 phase, D-cyclin interactors Cdk4 and Cdk6 (Fig. 1D and E). Cdk21 is approximately 
equally similar in amino acid sequence to both Cdk4 and Cdk6, with regions of high conservation within the kinase domain (51 and 52\% identical, respectively), whereas Cdk4 and Cdk6 are $66.44 \%$ identical to each other. We conclude that fishes possess an additional G1 Cdk beyond those found in mammals, Cdk21, with a previously undescribed function.

In order to determine if $c d k 21$ is expressed in the gonads of zebrafish and directly affects testis development, in situ hybridization (ISH) was performed using adult ovaries and testes, which contain germ cells at all stages of development from gonial cells to terminally differentiated gametes. The $c d k 21$ transcript was detected in gonads of both sexes, although was enriched in testes (Fig. 1G and I). Localization of cdk21 mRNA was detected in discrete clusters of spermatogonia, staged based on expression of vasa in cells of similar size and pattern (Fig. 1F and G). Oocytes of stages $\mathrm{Ib}$ and II exhibited faint $c d k 21$ expression in the cytoplasm and the Balbiani body, though it was not detected in pre-follicle stage oogonia or vitellogenic oocytes (Fig. 1I). Together, these results demonstrate that cdk21 transcript is enriched in spermatogonia, which undergo mitotic divisions, and thus may be a regulator of spermatogonial cell divisions.

\section{cdk21 mutants exhibit aberrant testicular germ cells and fail to undergo oogenesis}

To investigate the cellular defects resulting from cdk21 mutations, we assessed histology of testes from adult fish. Sexually mature cdk21 mutants homozygous for either allele (cdk2 $1^{\text {t30421 }}$ or $c d k 21^{u m b 7}$ ) or transheterozygous for both alleles had an abnormal composition of differentiating germ cells (Fig. 2). Normal adult zebrafish testes have tubules surrounded by a basement membrane that contain cysts of germ cells enclosed by Sertoli cells. Within the cysts, germ cells develop synchronously and are in the same stage of differentiation and the cell cycle: spermatogonia constitute the least differentiated cell type and are the mitotic population; spermatocytes are meiotic; spermatids are haploid and are terminally differentiating into sperm, which are located in the central lumen (Fig. 2A). Homozygous mutant $c d k 21$ testes had tubules containing cysts of germ cells, but with fewer germ cells of all types. The cdk21 mutant testes contained little or no sperm in contrast to wild type (Fig. 2B, C and D). In cdk21 mutants, germ cells often exhibited aberrant cell and nuclear morphology, as is common for cells with cell-cycle defects (Fig. 2 insets). While the histological defects in cdk21 mutant testes suggest that spermatogenesis may be defective at multiple stages, some germ cells overcame these defects and eventually differentiated into sperm.

To ask if mutant sperm were functional, we performed fertility assays by natural breeding. $67 \%$ of homozygous $c d k 21^{t 30421}$ were sub-fertile and the remaining 33\% were sterile (Table 1). Interestingly, fertile cdk2 $1^{t 30421}$ mutants typically became completely sterile following one or two breeding attempts (Table 1). Histological analysis of approximately $4 \mathrm{mpf}$ mutant males that had been bred to sterility indicated that germ cells were nearly or completely depleted demonstrating that $c d k 21$ is necessary for germ cell maintenance (Fig. 2F). By contrast, 1-year-old $c d k 21^{t 30421}$ mutant males that were raised in the absence of females maintained germ cells in similar abundance to younger mutant males, as assessed by histology (Fig. 2G). Therefore, the loss of germ cells observed following breeding was not due to age-related depletion, but rather a failure to replenish germ cells in direct response to breeding. Offspring resulting from outcrosses of cdk2 $21^{\text {t30421 }}$ mutant males were viable and did not exhibit embryonic defects, similar to outcrosses derived from $c d k 27^{t 30421}$ heterozygous fish. Thus, cdk21 mutant males have impaired fertility but are often able to make some functional sperm, however, cannot replenish sperm cells following breeding.

Table 1 Percentage of fertilized eggs resulting from breeding events between adult $c d k 21^{\text {t30412 }}$ homozygous mutant males $(n=12)$ and the average among wild-type siblings $(n=10)$.

\begin{tabular}{|c|c|c|c|c|c|}
\hline \multirow[b]{2}{*}{ Male genotype } & \multicolumn{5}{|c|}{ Breeding event, percent fertilized (number of eggs) } \\
\hline & 1 & 2 & 3 & 4 & 5 \\
\hline$c d k 21-/-1$ & $5.2 \%(380)$ & $0 \%(155)$ & $4.7 \%(105)$ & $0 \%(213)$ & $0 \%(104)$ \\
\hline$c d k 21-/-2$ & $0 \%(103)$ & $0 \%(202)$ & $0 \%(112)$ & $0 \%(419)$ & $0 \%(422)$ \\
\hline$c d k 21-/-3$ & $1.5 \%(335)$ & $0 \%(232)$ & $0 \%(307)$ & $0 \%(221)$ & $0 \%(101)$ \\
\hline$c d k 21-/-4$ & $0 \%(402)$ & $22 \%(286)$ & $0 \%(413)$ & $0 \%(187)$ & $0 \%(114)$ \\
\hline$c d k 21-/-5$ & $0 \%(347)$ & $0 \%(331)$ & $8.9 \%(290)$ & $0 \%(400)$ & $0 \%(118)$ \\
\hline$c d k 21-/-6$ & $0 \%(109)$ & $3 \%(197)$ & $0 \%(201)$ & $20 \%(420)$ & $0 \%(441)$ \\
\hline$c d k 21-/-7$ & $0 \%(231)$ & $0 \%(338)$ & 0\% (199) & $0 \%(220)$ & $0 \%(112)$ \\
\hline$c d k 21-/-8$ & $0 \%(110)$ & $0 \%(203)$ & $9.5 \%(188)$ & $0 \%(304)$ & $0 \%(102)$ \\
\hline$c d k 21-/-9$ & $0 \%(463)$ & $0 \%(196)$ & 0\% (103) & 0\% (291) & $0 \%(313)$ \\
\hline cdk21 -/- 10 & $0 \%(317)$ & $25 \%(257)$ & $0 \%(116)$ & $2.2 \%(178)$ & $0 \%(100)$ \\
\hline$c d k 21-/-11$ & $0 \%(125)$ & $0 \%(223)$ & $29 \%(212)$ & $0 \%(111)$ & $0 \%(212)$ \\
\hline$c d k 21-/-12$ & 0\% (192) & $0 \%(101)$ & 0\%(116) & $0 \%(487)$ & $0 \%(232)$ \\
\hline cdk21 +/+ (average) & $87.5 \%(231)$ & $75 \%(190)$ & $76 \%(191)$ & $74.4 \%(470)$ & $74.4 \%(430)$ \\
\hline
\end{tabular}

Only breeding events producing over 100 eggs were included. 
Cdk21 mutants were 100\% male (Fig. 2E), suggesting that $c d k 21$ is necessary for ovary differentiation, as is commonly seen from mutations or experiments disrupting germ cell development (see for example, Slanchev et al. 2005, Houwing et al. 2007, Siegfried \& Nüsslein-Volhard 2008, Rodríguez-Marí et al. 2011, Dranow et al. 2013, Saito et al. 2014, Tzung et al. 2015). To understand why loss of $c d k 21$ precludes female sex differentiation, gonads from juvenile fish were analyzed by histology at 3 weeks post fertilization (wpf). Juvenile zebrafish gonads undergo a pseudoovarian stage of development regardless of eventual sexual fate, characterized by the presence of stage $\mathrm{Ib}$ oocytes in addition to undifferentiated gonial cells (Fig. 2I). Interestingly, only one out of five juvenile cdk2 $1^{\text {t30421 }}$ mutants had oocyte-containing gonads at $3 \mathrm{wpf}$, in contrast to five of five heterozygous siblings (Fig. $2 \mathrm{I}$ and $\mathrm{J}$ ). We conclude that preclusion of female fate among cdk2 1 mutants owes to a defect or delay in the generation of oocytes in juvenile fish.

As cdk21 mutants displayed a distinct decrease in the number of sperm within the testis, it is possible that there was an increase in apoptotic clearance of cells. To determine if cdk21 mutant germ cells underwent increased apoptosis, we performed antibody labeling for cleaved Caspase-3. We observed a $2 \%$ average increase in apoptosis in mutant compared to wildtype testes (Fig. 2K). This moderate increase in apoptosis was statistically significant but biologically does not account for all of the morphologically abnormal cells and drastic reduction in sperm. We hypothesize that the majority of germ cells lacking cdk21 complete spermatogenesis, but may do so more slowly than normal. Alternatively, cells may die by non-cleaved Caspase-3-mediated mechanisms. Despite the abundant defects observed, functional sperm were produced by $c d k 21$ germ cells and mutant subfertility cannot be ascribed to the small increase in apoptosis alone.

\section{cdk21 is required for normal progression through spermatogenesis}

To determine whether the defects observed in cdk21 mutant testes resulted from cell cycle defects, we assayed proliferation and differentiation of germ cells. Progression through the cell cycle and timely differentiation of sperm was assayed by labeling DNA with BrdU, which incorporates into DNA during $S$ phase. Fish were incubated in water containing BrdU for $18 \mathrm{~h}$ followed by a chase period of either $16 \mathrm{~h}$ or 5 days in water without $\mathrm{BrdU}$. The former time period is predicted to label spermatogonia and primary spermatocytes, whereas the latter should label differentiated sperm (Leal et al. 2009). To compare any differences between sexually immature and mature testes, we performed BrdU labeling at two time points, approximately $2 \mathrm{mpf}$ (immature) and $4 \mathrm{mpf}$ (mature). Our analysis of these time points in wild-type and mutant animals found that testes from $2 \mathrm{mpf}$ animals had no or very few mature sperm while $4 \mathrm{mpf}$ animals had an abundance of sperm in the testis (Fig. 3A, B, C and D). After a 16-h chase, wild-type immature and mature testes typically exhibited BrdU labeling in primary spermatocytes, spermatogonia within cysts and single spermatogonia, as discerned by nuclear morphology (Fig. 3A and C). In both immature and mature cdk21 mutant testes, abundant spermatocytes were BrdU labeled, although nuclear morphology was often aberrant (Fig. 3E and G). After 5 days post chase, wild-type BrdU labeled cells had completed or nearly completed spermatogenesis (Fig. 3B and D). By contrast, mutants at either stage displayed little to no sperm (Fig. 3F and H). Additionally, mutant BrdU-labeled spermatocytes were still present and therefore were not progressing normally through spermatogenesis but were delayed or arrested in meiosis (Fig. 3F and $\mathrm{H}$ ). We counted BrdU-positive and -negative spermatogonia and spermatocytes and found no significant difference after the 16-h chase. After the 5 -day chase, we counted germ cells that had not yet exited meiosis (i.e. spermatogonia and spermatocytes) and found more BrdU-labeled cells present in mutant testes. These data indicate that, in mutants, many BrdUlabeled cells had not yet completed meiosis, whereas nearly all wild-type BrdU-labeled cells were postmeiotic (i.e. spermatids and spermatozoa) (Fig. 3l). Therefore, $c d k 21$ mutant cells did not progress through spermatogenesis at a normal rate. Furthermore, fewer BrdU-labeled spermatogonia were present in mutants than in wild type, demonstrating reduced spermatogonial proliferation (Fig. 3J). These data suggest a role for $c d k 21$ in spermatogonia proliferation as well as progression of meiosis in spermatocytes.

To further characterize the germ cell defects in $c d k 21$ mutant testes, protein expression of phospho-Histone $\mathrm{H} 3(\mathrm{pH} 3)$ was assayed by Western blot using whole testes lysates. $\mathrm{pH} 3$ labels cells in G2/M of mitosis and in the diplotene stage of meiosis I. Immature cdk21 mutant testes had reduced levels of $\mathrm{pH} 3$, whereas mature testis had increased levels (Supplementary Fig. 2). This supports our observations from BrdU assays of reduced spermatogonia proliferation in immature $c d k 21$ mutant testes. The increase in $\mathrm{pH} 3$ levels in mature mutant testes may indicate prolonged G2/M and/or diplotene stages. Overall, our results support the interpretation that loss of cdk21 affects proliferation and timely differentiation of male germ cells.

\section{cdk21 mutant testes have an overabundance of Sycp3}

To investigate defects or delays in the differentiation of primary spermatocytes in cdk21 mutants, we assessed synaptonemal complex protein 3 (Sycp3) in testes. Zebrafish primary spermatocytes have been shown to 


\section{Immature}
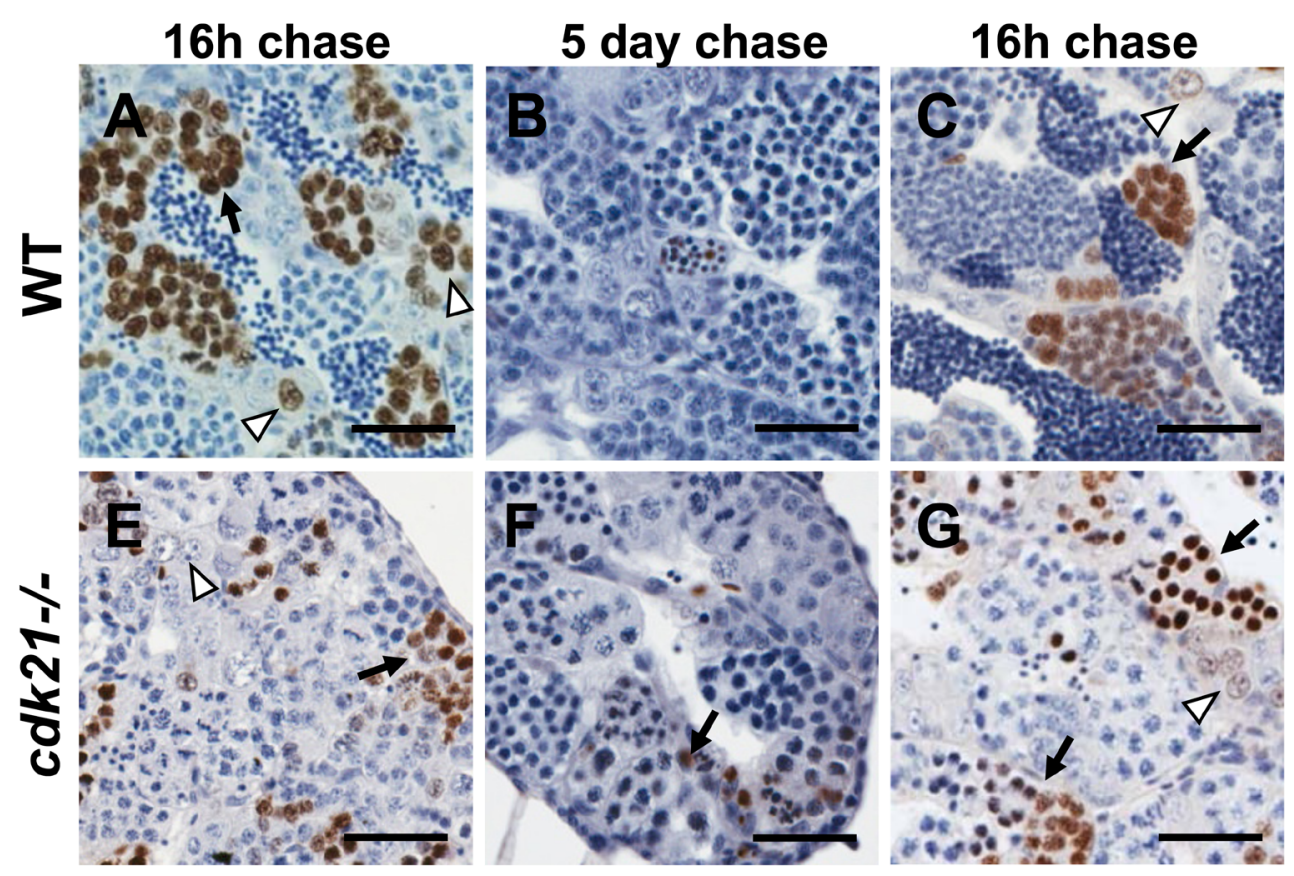

Mature
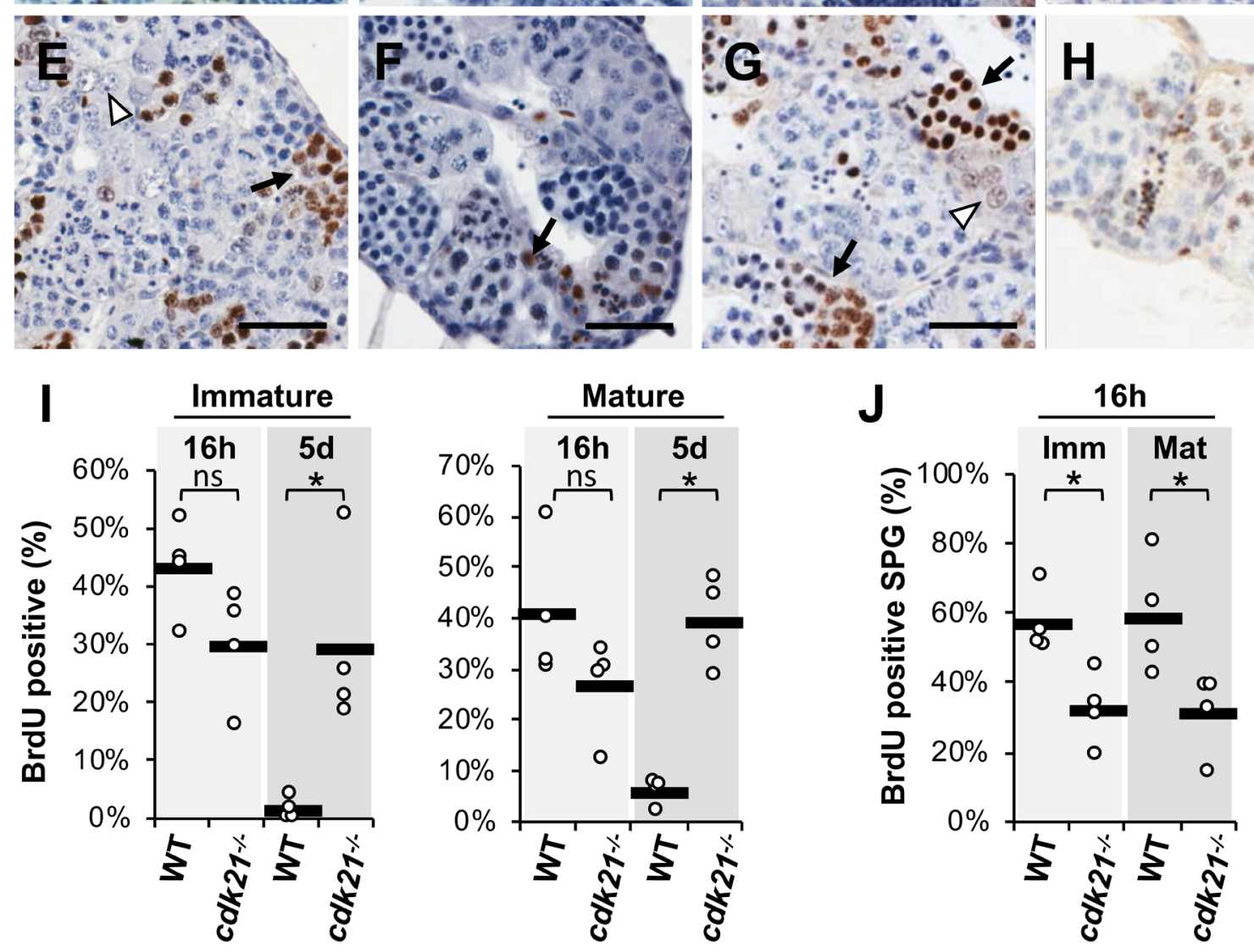

Figure 3 The $c d k 21$ gene is a regulator of proliferation and differentiation in male germ cells. (A, B, C, D, E, F, G and H) Immunohistochemistry of BrdU positive cells in testes from wild-type male zebrafish and $c d k 21^{t 30421}$ mutants exposed to BrdU for $18 \mathrm{~h}$ followed by either $16 \mathrm{~h}$ or 5 days (5 d) of no BrdU exposure (chase). (A and B) In immature ( 2 mpf) wild-type testes, BrdU marked spermatogonia and primary spermatocytes after the $16 \mathrm{~h}$ chase and spermatids after $5 \mathrm{~d}$ chase. (C and D) In mature ( $4 \mathrm{mpf})$ wild-type testes, BrdU labeled spermatogonia and primary spermatocytes after $16 \mathrm{~h}$ chase and spermatids/spermatozoa after $5 \mathrm{~d}$ chase. (E, F, G and H) Immature and mature cdk21 mutant testes had BrdU labeling in spermatogonia and spermatocytes after $16 \mathrm{hr}$ chase, and in primarily spermatocytes and spermatid/spermatozoa after $5 \mathrm{~d}$ chase. (I) Quantification of total BrdU positive cells (excluding post-meiotic cells). After $5 \mathrm{~d}$ chase, in wild-types very few BrdU labeled cells were spermatogonia or spermatocytes. In mutant testes, many BrdU labeled cells had not yet progressed to spermiogenesis in mutants $(P=0.03,5 \mathrm{~d}$ immature; $P=0.035 \mathrm{~d}$ mature). (J) Quantification of BrdU labeled spermatogonia after $16 \mathrm{~h}$ chase. The percentage of BrdU labeled spermatogonia out of total spermatogonia was calculated. Both immature and mature mutant testes had significantly fewer BrdU labeled spermatogonia ( $P=0.03$; immature; $P=0.03$ mature). Horizontal black bars on all charts denote the mean. White arrowhead, spermatogonia; arrow, spermatocytes; WT, wild-type; ns, not significant. Scale bar $=20$ microns.

display canonical patterns of Sycp3 localization during the stages of meiotic prophase I (Saito et al. 2011). To examine the dynamics of meiotic prophase in $c d k 21$ mutant testes, Sycp3 protein levels were detected by Western blot and localization by immunofluorescence (IF) (Fig. 4). Surprisingly, levels of Sycp3 protein were 

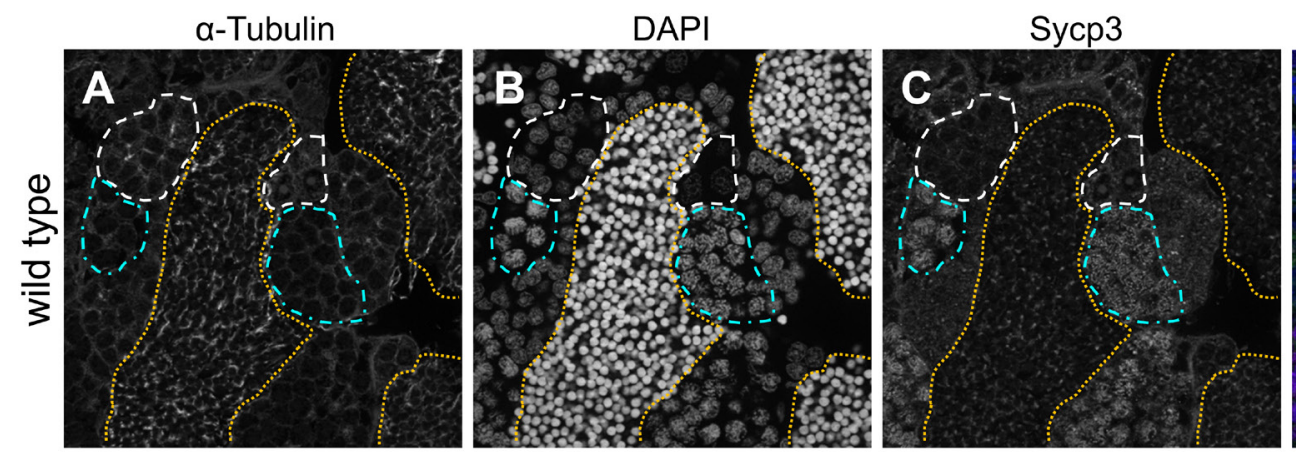

a-Tubulin DAPI Sycp3
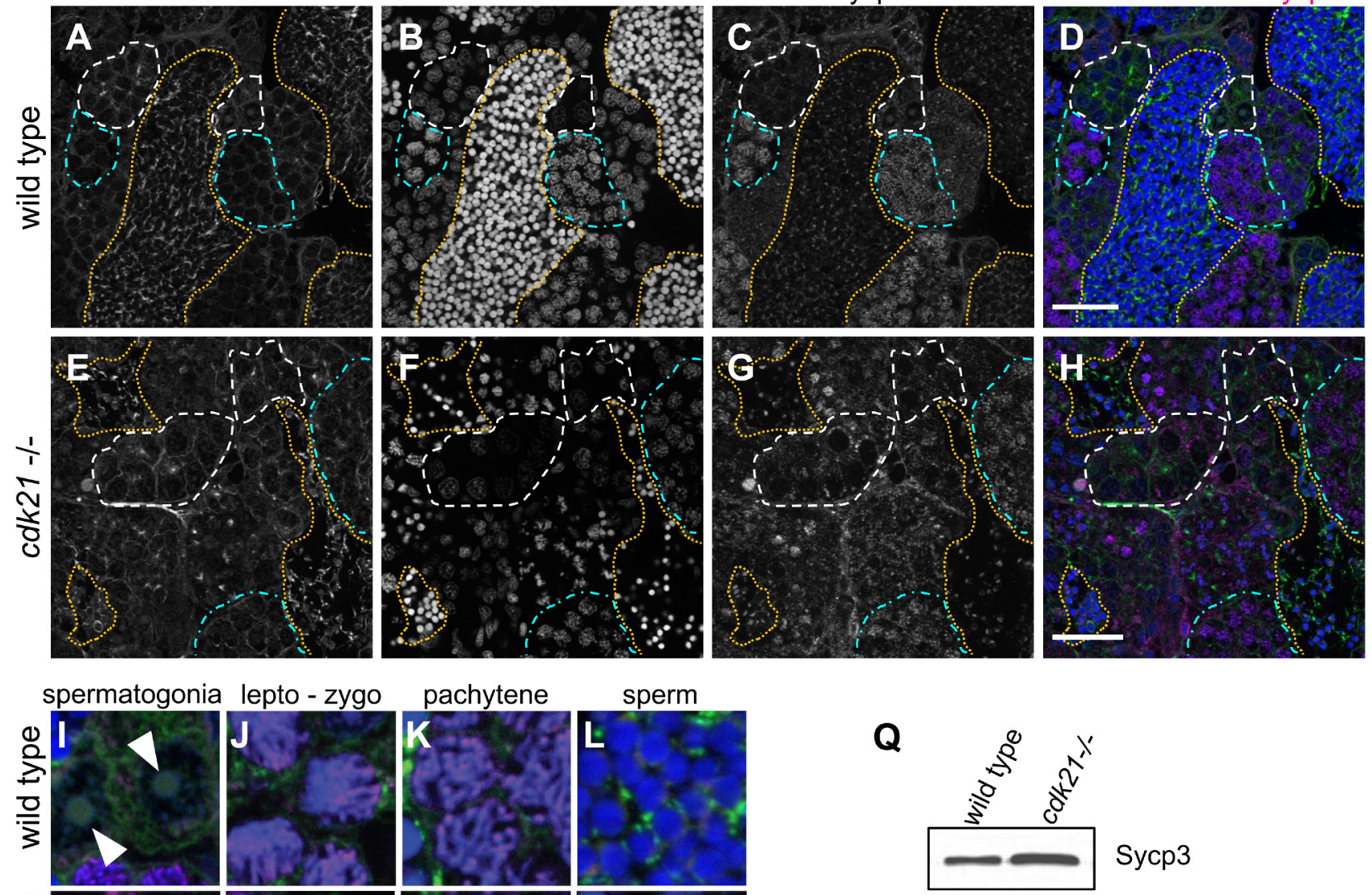

\section{sperm}
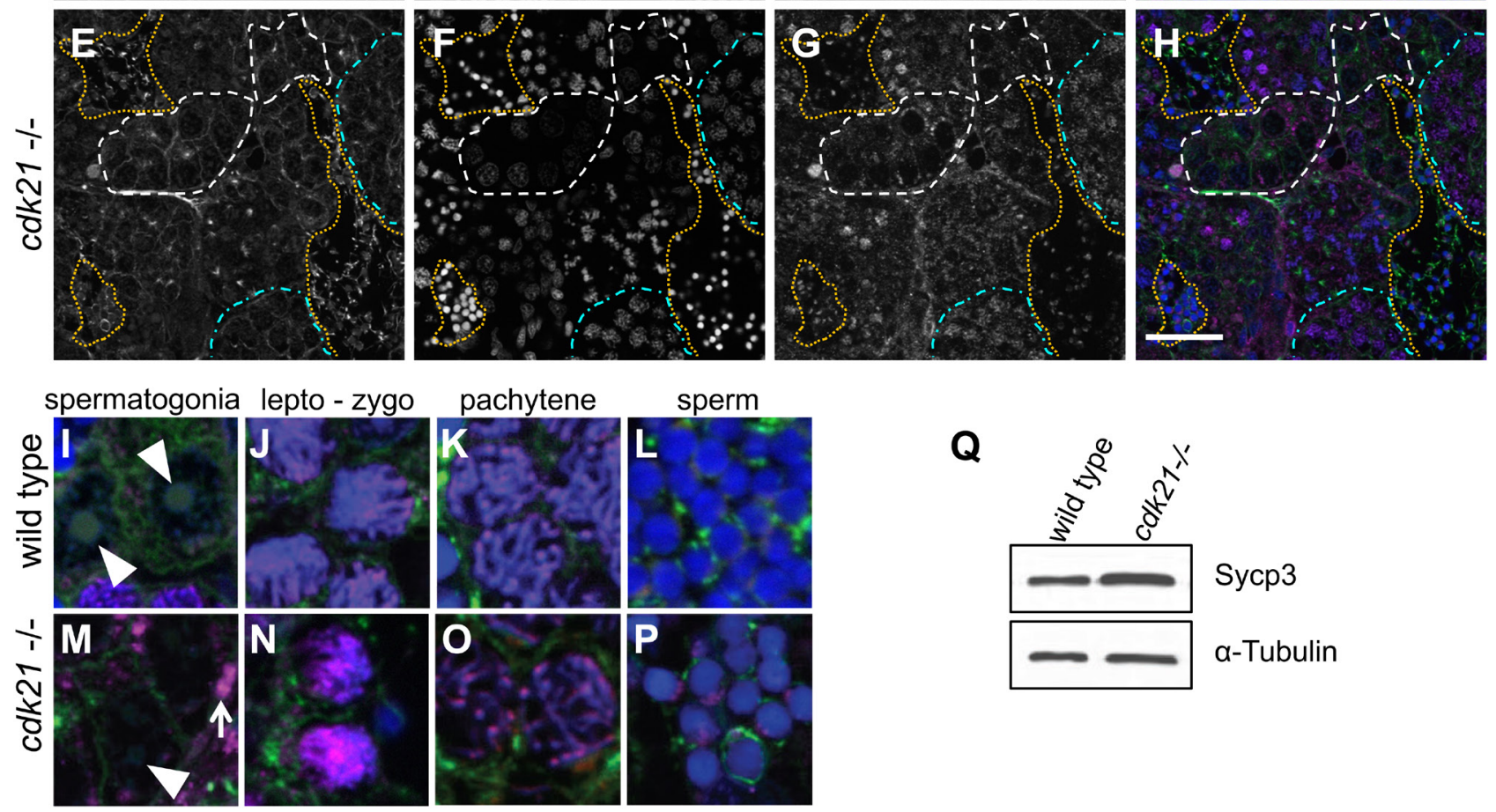

Figure 4 cdk21 mutant germ cells exhibit ectopic expression of Sycp3. (A, B, C, D, E, F, G, H, I, J, K, L, M, N, O and P) Immunofluorescence on sections of adult testes. Wild-type (A, B, C and D) and $c d k 21^{\text {t30421 }}$ mutant (E, F, G and H) testes labeled for $\alpha$-Tubulin, DNA (DAPI), and Sycp3. Examples of germ cells cysts containing spermatogonia (white dashed), primary spermatocytes (turquoise dash-dotted), and spermatozoa (orange dotted) are outlined. (I, J, K, L, M, N, O and P) High magnification of testicular germ cells showing localization of $\alpha$-Tubulin (green), DNA (blue) and Sycp3 (magenta). (I and M) Early stage spermatogonia have relatively uncondensed DNA and prominent nucleoli (arrowhead). Wild-type spermatogonia (I) have little to no detectible Sycp3, whereas cdk21 mutant spermatogonia (M) frequently had cytoplasmic Sycp3 that often aggregated in puncta (arrow). Wild-type ( and $\mathrm{K}$ ) and mutant ( $\mathrm{N}$ and $\mathrm{O}$ ) spermatocytes exhibited Sycp3 associated with chromosomes. Mutant sperm (P) displayed a pronounced Sycp3 compared to wild-type sperm (L). (Q) Western blots of protein lysates extracted from zebrafish testes. Sycp3 was more abundant in mutant testes than wild-type. Tubulin was used as a loading control. Lepto-zygo, leptotene to zygotene stage spermatocytes; White arrowhead, nucleoli; white arrow, cytoplasmic Sycp3 puncta. Scale bar=20 microns.

elevated in mutant compared to wild-type testes (Fig. 4Q and Supplementary Fig. 2). Analysis by IF revealed that increased Sycp3 did not merely reflect an overabundance of spermatocytes in mutant testes, but rather ectopic expression of this protein throughout the germ cells (Fig. 4A, B, C, D, E, F, G, H, I, J, K, L, $\mathrm{M}, \mathrm{N}, \mathrm{O}$ and $\mathrm{P}$ ). In wild type, only spermatocytes from leptotene through pachytene stage showed punctate to threadlike Sycp3 which was primarily associated with DNA (Fig. 4A, B, C and D, I, J, H, K and L). In cdk21 mutants, Sycp3 localized normally to the synaptonemal complex during meiotic prophase I (Fig. 4E, F, G and H, $\mathrm{M}, \mathrm{N}, \mathrm{O}$ and P). However, spermatogonial cells lacking cdk21 accumulated Sycp3 precociously in perinuclear domains and cytoplasm, and in post-meiotic sperm Sycp3 was more prominently retained. These findings suggest that $c d k 21$ is necessary to prevent precocious expression of meiotic genes in spermatogonia and prevent prolonged expression or protein clearance beyond meiosis. 


\section{G1 CDKs are differentially expressed across developmental stages and tissue types}

To ask whether G1 Cdks have specialized functions in differentiated zebrafish tissues, cdk4, cdk6 and cdk21 transcripts were analyzed in whole organs from adult fish using RT-PCR (Fig. 5A). All three genes were expressed in gonads of both sexes, although cdk4 appeared more prominent in ovaries and cdk21 more prominent in testes. Overlapping expression of these genes was observed for several tissues. Outside of the ovary, cdk4 expression was very weakly or not detected and may point to a more specialized role for this gene in ovaries.

Expression of $c d k 4, c d k 6$ and $c d k 21$ were also assayed in developing zebrafish from $24 \mathrm{~h}$ post fertilization (hpf) to 3 weeks post fertilization (wpf), the latter of which is the approximate time point when the gonad begins to exhibit histological differences indicative of testis or ovary differentiation. For stages $1-3 \mathrm{wpf}$, only torsos spanning the swim bladder length were collected for
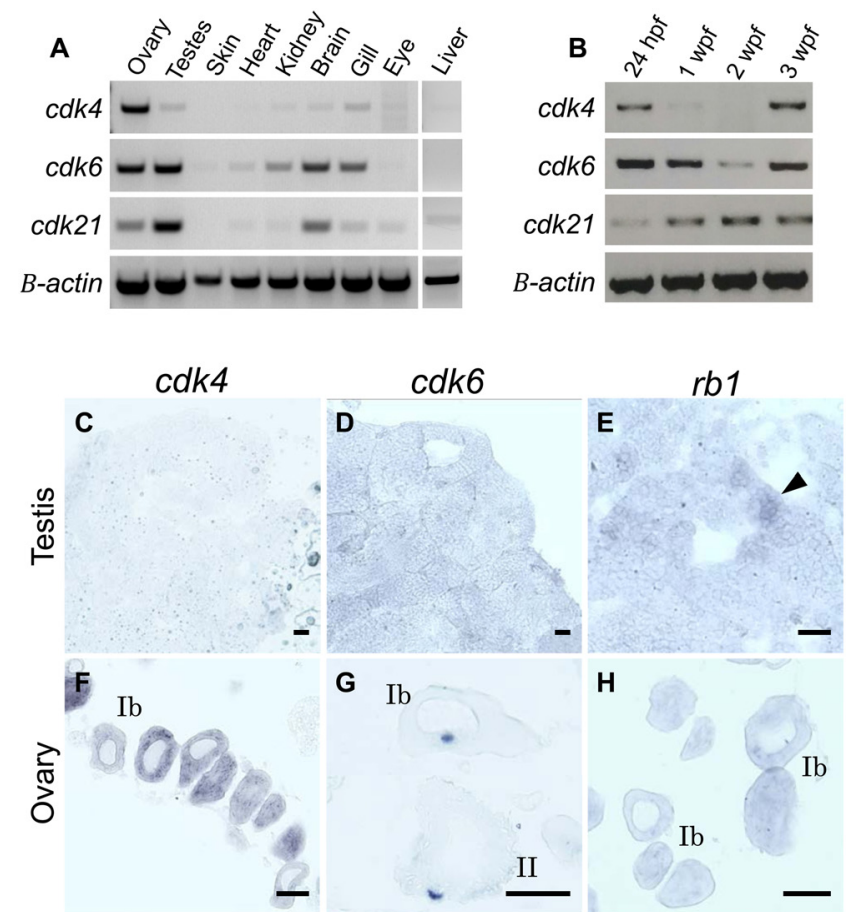

Figure 5 The G1 cyclin-dependent kinases have distinct expression patters. (A and B) RT-PCR from zebrafish tissues. (A) mRNA expression of $c d k 4, c d k 6$ and $c d k 21$ in tissues from adult fish. (B) mRNAs expression in whole $24 \mathrm{hpf}$ embryos, or torsos of $1 \mathrm{wpf}$, 2 wpf, and 3 wpf zebrafish. (C, D, E, F, G and H) Section in situ hybridizations of adult testes and ovaries. (C and F) cdk4 transcript was not detectable in the testes but localized prominently to the cytoplasm of stage Ib oocytes. (D and G) cdk6 was detected uniformly throughout the testes, but in oocytes was restricted to the Balbiani body in stage Ib oocytes and the vegetal cortex of stage II oocytes. (E) The rb1 transcript was expressed throughout the testes but was enriched in spermatogonia. $(\mathrm{H})$ In ovaries, $r b 1$ transcript was localized to the cytoplasm of stages Ib oocytes. Oocyte staging according to Maack \& Segner 2003. Scale bars $=20$ microns.
RT-PCR. Similar to the whole tissue analysis, $c d k 4$, cdk6 and $c d k 21$ were found to have distinct patterns of expression across developmental stages (Fig. 5B). Expression of $c d k 21$ appeared to increase with age, and thus with gonad differentiation, whereas cdk6 was expressed across all time points. Uniquely, cdk4 expression was primarily detected at $24 \mathrm{hpf}$ and $3 \mathrm{wpf}$, suggesting a specific role in embryogenesis and perhaps the juvenile ovary stage of gonad development, as it was also enriched in adult ovaries. Taken together, these results demonstrate the diversity of spatiotemporal expression of these G1 CDKs and underscore the likelihood that they have unique functions.

As expression of $c d k 4,6$ and 21 were all detected in adult gonads, we sought to determine gonadal cell subtype localization of these transcripts, via section ISH. Expression of $r b 1$ was also analyzed given its functional relationship to $c d k 4$ and $c d k 6$. By ISH, cdk4 and rb 1 were found in the cytoplasm of stage I and II oocytes (Fig. 5F and H). Localization of $c d k 6$ was tightly restricted to the Balbiani body in stage Ib oocytes and remained sequestered at the vegetal pole through stage II (Fig. 5G). In the testes, $c d k 4$ and $c d k 6$ were present diffusely across cell types, although expression of the former was barely detectable (Fig. 5C and D). In contrast, $r b 1$ was enriched in spermatogonia (Fig. 5E). Thus, cdk21 (Fig. 1G) and $r b 1$ were both enriched in spermatogonial cells in the testis, whereas $c d k 4$ and $c d k 6$ were not enriched or specific to a particular testicular cell type.

\section{cdk4, cdk6 and cdk21 have unique and redundant functions in zebrafish}

In order to elucidate the redundancy or novelty of the function of $c d k 4, c d k 6$ and $c d k 21$, mutant alleles causing deletions and frame shifts within the kinase domain were isolated for each gene using CRISPR/Cas9 genome editing (Supplementary Fig. 1B and D).

Homozygous cdk4 mutants were adult viable with little observable defects. However, all cdk4 mutants were male $(n>50)$ similar to the effect seen in $c d k 21$ mutants. Due to the pronounced expression of $c d k 4$ in oocytes (Fig. 5), loss of female development may reflect a requirement for $c d k 4$ in normal oogenesis or ovary development. Interestingly, $c d k 4$ mutant males had poor breeding success and low fertility (Table 2). Mutant males only induced wild-type females to breed 1 or 2 times of 3 total pairings, compared to $100 \%$ breeding success for pairings with heterozygous males (Table 2). Furthermore, when breeding was successful, females produced fewer eggs and fertilization rates were lower when paired with mutant than with heterozygous males. Progeny from mutant sperm survived and appeared normal up to 5 days post fertilization (dpf); later stages were not analyzed. We next investigated histology of cdk4 mutant testes (Fig. 6). Surprisingly, cdk4 mutant testes displayed an overabundance of sperm relative to 
Table 2 Mutant $c d k 4$ mutant males have poor breeding capacity and fertility.

\begin{tabular}{lccc}
\hline \multirow{2}{*}{ Male genotype } & \multicolumn{3}{c}{ Breeding attempt, \# eggs (\% fertilized) } \\
\cline { 2 - 4 }$c d k 4-/-1$ & 1 & 2 & 3 \\
$c d k 4-/-2$ & 0 & 0 & $117(76 \%)$ \\
$c d k 4-/-3$ & $38(26 \%)$ & 0 & 0 \\
$c d k 4-/-4$ & 0 & $17(65 \%)$ & $33(0)$ \\
$c d k 4-/-5$ & $33(82 \%)$ & 0 & $10(80 \%)$ \\
$c d k 21+/-1$ & 0 & $\mathrm{NC}(0)$ & 0 \\
$c d k 21+/-2$ & $150(99 \%)$ & $300(97 \%)$ & $208(79 \%)$ \\
$c d k 21+/-3$ & $22(100 \%)$ & $120(86 \%)$ & $142(94 \%)$ \\
\hline
\end{tabular}

Five adult $c d k 4^{u m b 8}$ mutant males of 5-6 months post fertilization paired with a wild-type female showed poor breeding success. Mutant males induced egg production by females significantly fewer times than heterozygous males $(P=0.007)$. When breeding was successful, females paired with mutants produced fewer eggs $(P=0.016)$. Fertilization rates of mutant males was also lower than heterozygotes $(P=0.003)$. All females used for a particular breeding attempt were from the same population of fish. $P$ values were calculated using the Mann-Whitney test for unpaired data. NC, not counted.

wild-type males but exhibited otherwise histologically normal-appearing testes (Fig. 6A, B and C). Because cdk4 mutants produced viable sperm but did not reproduce well by natural breeding, $c d k 4$ may be needed for normal courtship behaviors or for sperm release during mating.

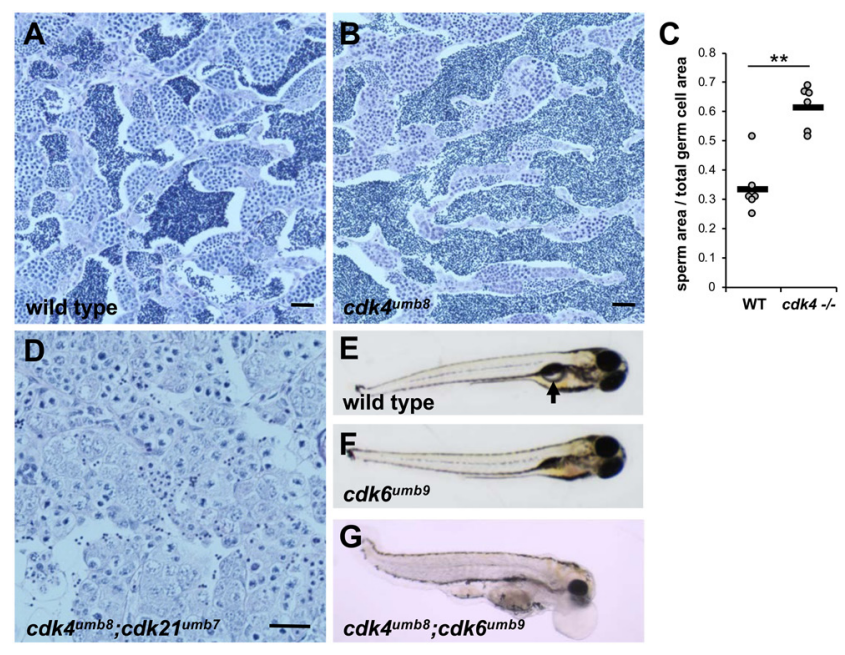

Figure $6 c d k 4$ and $c d k 6$ mutant phenotypes do not resemble loss of cdk21. (A, B and D) H\&E stained sections of adult testes. (A) Wild-type adult testes. (B) $c d k 4^{u m b s}$ mutants had histologically normal testes except for overabundance of sperm. (C) Quantitation of the area occupied by sperm/the total area occupied by all germ cells (including sperm) from histological sections; the black bar shows the mean ( $N=6$ individuals per genotype, $P=0.002)$. (D) $c d k 21^{t 30421} ; c d k$ $4^{u m b 8}$ double mutant testes resembles that of $c d k 21^{\text {t30421 }}$ single mutants. (E) Wild-type larva at $5 \mathrm{dpf}$ showing an inflated swim bladder (arrow). (F) $c d k 6^{u m b 9}$ at $5 \mathrm{dpf}$ showing lack of swim bladder. (G) $c d k 4^{u m b 8} ; c d k 6^{u m b 9}$ double mutants displayed a range of severe developmental defects by $5 \mathrm{dpf}$, including short/wide body, lack of swim bladder, craniofacial deformities and edemas. Scale bar $=20$ microns.
Double $c d k 4 ; c d k 21$ mutants were generated to determine if these two mutations genetically interact in testis development. The $c d k 4 ; c d k 21$ double mutant testes closely resembled testes of cdk21 single mutants with reduced sperm counts and abundance of cells with abnormal nuclear morphology (Fig. 6D). Furthermore, cdk4;cdk21 double mutant males bred with similar success to wild-type siblings, as was true for cdk21 single mutants $(n=3$ double mutant males paired with wild-type females, three breeding events each with at least $80 \%$ fertilization of $>100$ deposited eggs). Strikingly, only $15 \%$ of progeny from $c d k 4 ; c d k 21$ mutant males survived up to $5 \mathrm{dpf}$, with death occurring after embryogenesis, typically between $24 \mathrm{hpf}$ and $5 \mathrm{dpf}$ ( $n=8$ clutches from 3 mutant males paired with wild-type females, clutch sizes were 98-230 deposited eggs). These data demonstrate that $c d k 21$ is epistatic to $c d k 4$ with respect to both spermatogenesis and breeding success. However, while both $c d k 21$ and $c d k 4$ are dispensable for zebrafish embryonic/larval development, there is a paternal requirement to have at least one functional copy of $c d k 4$ or $c d k 21$ to produce viable offspring.

Loss-of-function cdk6 mutants displayed developmental defects in larvae. By $5 \mathrm{dpf}$, cdk6 mutants exhibited lack of swim bladder but appeared otherwise normal (Fig. 6F). By $2 \mathrm{wpf}, \mathrm{cdk} 6$ mutant survival was on average only $4 \%$ and no mutants survived to adulthood ( $n=96$, from 4 clutches). Double $c d k 4 ; c d k 6$ mutants were larval lethal and exhibited more severe developmental defects than cdk6 single mutants, including gross morphological abnormalities and edema formation (Fig. 6G). The cdk6;cdk21 double mutants were not adult viable suggesting embryonic or larval lethality, although severity of this phenotype relative to cdk6 single mutants was not investigated. Taken together, these data suggest that cdk6 is indispensable across several tissue types and developmental stages in zebrafish. Furthermore, cdk 4 and $c d k 6$ are partially redundant in embryogenesis.

\section{Discussion}

Here, we described the function of $c d k 21$, a novel cell cycle regulator in germ cells of zebrafish. Homology of Cdk21 to Cdk4 and Cdk6 suggests that this protein functions similarly to these canonical G1 phase kinases, which regulate progression through G1 of the cell cycle. Zebrafish cdk21 mutants were viable but were exclusively male with impaired fertility. We found that cdk21 was necessary for germ cell maintenance, mitotic expansion of spermatogonia and timely progression through meiosis in testicular germ cells. The culmination of these defects resulted in severely reduced sperm and subfertility/sterility. Furthermore, we characterized the expression and function of cdk4 and cdk6 in zebrafish and found that these three kinases have specific and some overlapping functions. To our knowledge, this is 
the first study of the $c d k 21$ gene in any organism and the first functional analysis of $c d k 4$ and $c d k 6$ in zebrafish. We show that cdk21 has a specific role in regulating germ cell differentiation through mediating the cell cycle in germ cells.

\section{cdk21 regulation of spermatogonia proliferation and maintenance}

Zebrafish harboring cdk21 mutations exhibited reduced spermatogonial proliferation and failure to maintain the germline stem cell population following breeding. Expression of cdk21 transcript in spermatogonia and decreased spermatogonia proliferation in mutants suggests that $c d k 21$ functions in a progenitor-supporting capacity by promoting spermatogonia proliferation. Additionally, cdk21 mutant males that had undergone breeding failed to maintain germ cells pointing to a failure in spermatogonial stem cell maintenance. CDK4/6 family proteins have known roles in regulating and maintaining stem cell populations. For example, in hematopoietic stem cells (HSCs), CDK6 is thought to 'prime' short-term HSCs (ST-HSCs) for quicker exit from quiescence compared to long-term HSCs (LT-HSCs), which do not express CDK6 (Laurenti et al. 2015). This mechanism is thought to be important for rapid responses to stem cell activation by ST-HSCs in response to mitogenic signals, whereas LT-HSCs exit quiescence more slowly, thereby maintaining the stem cell pool. This dichotomy allows a rapid activation of stem cells when needed without depleting the stem cell population. cdk21 mutant zebrafish maintained their germ cells when raised in the absence of females, however, exhibited germ cell depletion and became infertile after multiple breeding encounters. Therefore, similar to Cdk6 in HSCs, cdk21 is necessary to maintain a balance between replenishing the sperm cells when sperm production is at high demand and preserving the germline stem cells.

In mouse the CDK4/6 kinase target RB1 regulates spermatogonia proliferation and the self-renewal capacity of the spermatogonial stem cells (Hu et al. 2013, Yang et al. 2013). Zebrafish $c d k 21$ and $r b 1$ expression were both enriched in spermatogonia; therefore, Rb1 is a potential kinase target of Cdk21 in these cells, although further biochemical experiments are needed to test this. In mouse, conditional mutations disrupting $R b 1$ in prospermatogonia led to germline depletion by 2 months, resulting from a failure in stem cell selfrenewal. However, prior to germ cell loss, an increase in spermatogonial proliferation was observed, which is typical in cells deficient for $R b 1$ due to unregulated proliferation (Hu et al. 2013, Yang et al. 2013). CDK4/6 proteins typically repress RB1 thereby mediating controlled progression through G1 and into S phase. Thus, loss of CDK4/6 function often causes slowed cell proliferation. The decreased spermatogonia proliferation and hypoplastic testis in zebrafish cdk21 mutants fits with typical phenotypes for loss of $C d k 4 / 6$ function. It is therefore plausible that $\mathrm{Cdk} 21$ regulates cell proliferation in zebrafish spermatogonia by modulating $\mathrm{Rb} 1$, as is the canonical role of CDK4/6 in cell cycle regulation in many cell types.

Zebrafish cdk21 mutants exhibited a similar failure to maintain a germline stem cell population as seen in $R b 1$ mutant mice, although only in situations where sperm production was at high demand - after frequent breeding. Unlike the antagonistic relationship between CKD4/6 and RB1 during cell cycle regulation, in spermatogonial stem cells, both Cdk21 and Rb1 seem to similarly promote stem cell self-renewal. Because mouse $R b 1$ and zebrafish $c d k 21$ both are necessary for spermatogonial stem cell renewal, it is worth investigating if zebrafish Cdk21 confers adequate stem cell self-renewal through Rb1 regulation.

\section{Cdk21 is necessary for progression through meiosis}

In addition to defects in spermatogonia, cdk21 mutants failed to progress through meiosis normally. BrdUlabeling experiments revealed prolonged meiosis in cdk21 mutant testes. CDK proteins may have unique functions in meiotic prophase, for example in mammals, CDK4 localized to recombination nodules on synapsed chromosomes (Ashley et al. 2001). Thus, this class of CDKs likely have roles in synapsis or recombination during meiosis that are unrelated to their canonical role in cell cycle regulation. Interestingly, cdk21 transcripts were not detectable by ISH in meiotic germ cells. Cdk21 protein may be present in spermatocytes where it has a role in meiosis or the prolonged meiosis may be caused by defects carried over from prior proliferative stages.

Several meiosis-defective zebrafish mutants have been characterized previously; however, all were completely sterile and did not complete spermatogenesis. The ietsugu (its), iesada (isa) and iemochi (imo) mutants arrested specifically at either preleptotene (its) or zygotene stages (isa and imo) exhibited increased apoptosis and failed to complete spermatogenesis (Saito et al. 2011). The molecular characterization of these mutations has not yet been reported. Male zebrafish with a loss-offunction mutation in the DNA mismatch repair gene MutL Homolog 1 ( $\mathrm{m} / \mathrm{h} 1)$ arrested in meiotic prophase, but unlike cdk21 mutants, they failed to complete meiosis and produce sperm (Feitsma et al. 2007). The cdk21 mutants, described here, did not exhibit a block at a specific stage of spermatogenesis, but instead had defects at multiple stages; yet, some germ cells were able to complete spermatogenesis.

Cdk21 was necessary for spermatocyte-restricted expression of Sycp3 protein. Our analysis of Sycp3 in $c d k 21$ mutant testes revealed an unusual overabundance of Sycp3 protein, which is normally limited to meiotic prophase where it is a component of the synaptonemal 
complex. Sycp3 protein was found in all stages of spermatogenesis in cdk21 mutant testes, from spermatogonia through spermatozoa. This observation indicates that $c d k 21$ is necessary for spatial restriction of SC components to prophase I. As CDK4 and CDK6 are both known to directly (CDK6) or indirectly (CDK4 and 6) regulate transcription, it is feasible that $\mathrm{Cdk} 21$ restricts appropriate expression of Sycp3 to meiotic prophase.

Surprisingly, the meiotic defects in cdk21 mutants appear to have little-to-no consequences for the few gametes that are produced. Mutant sperm are capable of fertilizing eggs, which develop as normal embryos and adults with typical sex ratios (when not homozygous for cdk21 loss). This points to the ability for other Cdks to partially compensate for delays in both the mitotic and meiotic stages of germ cell differentiation. As very few sperm cells are made, and only modest elevation in cleaved Caspase-3-positive cells are observed, it is likely that many cdk21 mutant germ cells fail to adequately complete mitosis and/or meiosis and undergo senescence or non-Caspase-3-mediated cell death.

\section{cdk21 is necessary for timely production of female- promoting oocytes in juveniles}

The role of $c d k 21$ in germ cell differentiation likely extends to the undifferentiated gonad, which is yet to exhibit morphologically differentiated sex. Loss of cdk21 function resulted in exclusively male sexual fate, which is likely due to germ cell defects in juvenile fish as female sexual fate is dependent on the presence of germ cells and sustained meiotic oocytes after the juvenile ovary stage (Hsu et al. 2018). We found that cdk21 mutant gonads largely do not exhibit a juvenile ovary stage, although they do not develop testes earlier than wild-type fish. As there is relatively little expression of cdk21 in adult ovaries compared to testes, we do not attribute this lack of feminization to a necessity of $c d k 21$ for female fate, but rather as a secondary consequence of defects in germ cells. Timing of cell proliferation transition into meiosis, and induction of female germ cell apoptosis all shape sexual outcome, and these processes are mediated in part by cell cycle regulators. We hypothesize that loss of cdk21 function precludes female fate indirectly by affecting timely generation of female-supporting oocytes in the undifferentiated gonad.

\section{Cdk4, 6 and 21 fulfill unique and partially overlapping roles in zebrafish embryogenesis and gonads}

We have demonstrated here unique and redundant roles for the three G1 Cdks present in fishes, cdk4, cdk6 and cdk21. We found distinct mRNA expression patterns of these three genes and found that cdk4 transcripts were enriched in ovaries, whereas cdk21 transcripts were enriched in testes suggesting sex-specific roles in gamete development. Mouse knockouts of $C d k 4$ displayed impaired fertility and spermatogenesis defects; however, somatic testicular cells were also affected making it difficult to parse the precise function of $C d k 4$ in germ cells (Rane et al. 1999, Tsutsui et al. 1999, Sherr \& Roberts 2004). By contrast, we found that zebrafish cdk4 mutants had relatively normal spermatogenesis, with the exception of sperm overabundance, whereas cdk21 mutants had severe defects specific to germ cells in the testis. Thus, $c d k 21$, rather than $c d k 4$, is essential for spermatogenesis in zebrafish.

Mice null for Cdk4 or Cdk6 are viable (although viability is reduced in Cdk4 mutants), which is a departure from the larval lethal phenotype we report in zebrafish cdk6 mutants (Rane et al. 1999, Tsutsui et al. 1999, Malumbres et al. 2004). This suggests that cdk6 has adopted a more essential and global role in zebrafish, and thus has diverged significantly in function from the mammalian Cdk6. In contrast, zebrafish cdk4 mutants had no apparent defects other than the absence of female fate, overabundance of sperm and poor breeding success. These observations indicate that $c d k 4$ has a mostly gonadal/reproductive function in zebrafish and perhaps is necessary for oogenesis, as suggested by robust expression of $c d k 4$ in oocytes and inability of mutants to specify or maintain female fate.

Double mutant analysis of the G1 cdks in zebrafish revealed some additional defects to those observed in single mutants. Double mutants of zebrafish $c d k 4$ and cdk6 exhibited more severe embryonic/larval defects than either single mutant, similar to mouse knockout models (Malumbres et al. 2004). Early lethality was observed in both zebrafish and mice, and in mice, lethality was shown to result from overall underdevelopment and multilineage failures. Thus, $c d k 4$ and $c d k 6$ have partially redundant functions in both mice and zebrafish. We did not determine the cause of larval death in our mutants but future analysis of individual tissues will be informative in parsing the precise role of these important cell cycle regulators during embryonic and larval development in zebrafish. Double mutants of $c d k 4$ and $c d k 21$ were adult viable and exhibited similar gonadal defects to $c d k 21$ mutants indicating that these genes are not acting redundantly in testicular germ cell development. Interestingly, $c d k 4 ; c d k 21$ double mutants exhibited paternal-effect lethality that was not apparent in either single mutant. Therefore, these two genes may act to regulate components in sperm that are paternally provided to the embryo, such as centrioles or miRNAs or sperm produced by these double mutant males may be aneuploid causing aberrant development in offspring. Overall, double mutant analysis revealed redundancy in $c d k 4$ and cdk6 during embryo/larval development in zebrafish; however, cdk4 and cdk21 had largely nonoverlapping functions. 


\section{Conclusions}

Here, we have demonstrated non-overlapping functions of the zebrafish G1 CDKs, Cdk4, 6 and 21 and identified a novel regulator of the cell cycle in the male germline of fish - Cdk21. Teleost fishes possess three genes of the cdk4/6 gene family in contrast to the two described in tetrapods. The zebrafish $c d k 21$ gene appears to control three aspects of testis function: continued renewal of germ cells following breeding, spermatogonia proliferation (mitosis) and progression through meiosis. Mammalian loss-of-function studies suggest $C d k 4$ is essential in several cell types of the testis, while Cdk6 is dispensable. We have shown that these roles are not conserved in zebrafish, where $c d k 4$ affects reproduction but is non-essential for the testis and cdk6 is required for larval survival. As cdk21 is present in lineages ancestral to both teleosts and tetrapods and therefore was most likely lost in the tetrapod lineage, we postulate that $C d k 4$ and $C d k 6$ have evolved to carry out roles in tetrapods that were originally controlled by $c d k 21$. It will be important to characterize the role of $c d k 21$ in other fish species in order to assess the diversity of its function. In the context of plastic and highly divergent reproductive strategies across fishes, genes such as $c d k 21$ may enable flexibility in terms of sexual fate and variations in breeding cycles.

\section{Supplementary data}

This is linked to the online version of the paper at https://doi.org/10.1530/REP-18-0386.

\section{Declaration of interest}

The authors declare that there is no conflict of interest that could be perceived as prejudicing the impartiality of the research reported.

\section{Funding}

$\mathrm{K} \mathrm{R} \mathrm{S}$ and $\mathrm{K} \mathrm{A} \mathrm{W}$ were supported by the UMB-DF/HCC U54 Partnership, NIH/NCl 1U54CA156734, and K A W was supported by a Sanofi Genzyme/Oracle Graduate Research Fellowship and a Doctoral Dissertation Fellowship from UMass Boston. A N was supported by the UMB Initiative for Maximizing Student Development program, NIH R25GM076321.

\section{Acknowledgements}

The authors are grateful to the three anonymous reviewers for their thorough and thoughtful comments that have greatly improved this manuscript. They thank Jaclyn Grenier and Cristina Rivera for technical contributions to this study. They thank Dana-Farber/Harvard Cancer Center in Boston, MA, for the use of the Specialized Histopathology Core, which provided slide scanning services, supported in part by an $\mathrm{NCl}$ Cancer Center Support Grant, NIH 5 P30 CA06516. The screen in which the $c d k 21^{t 30421}$ mutant was isolated was supported by the European Commissions, ZF-MODELS 6th framework program (EC Contract LSHG-CT-2003-503496).

\section{References}

Anderson JL, Marí A, Braasch I, Amores A, Hohenlohe P, Batzel P \& Postlethwait JH 2012 Multiple sex-associated regions and a putative sex chromosome in zebrafish revealed by RAD mapping and population genomics. PLOS ONE 7 e40701. (https://doi.org/10.1371/journal. pone.0040701)

Ashley T, Walpita D \& de Rooij DG 2001 Localization of two mammalian cyclin dependent kinases during mammalian meiosis. Journal of Cell Science 114 685-693.

Bowen ME, Henke K, Siegfried KR, Warman ML \& Harris MP 2012 Efficient mapping and cloning of mutations in zebrafish by low-coverage wholegenome sequencing. Genetics 190 1017-1024. (https://doi.org/10.1534/ genetics.111.136069)

Bradley KM, Breyer JP, Melville DB, Broman KW, Knapik EW \& Smith JR 2011 An SNP-based linkage map for zebrafish reveals sex determination loci. G3 1 3-9. (https://doi.org/10.1534/g3.111.000190)

Daane JM, Rohner N, Konstantinidis P, Djuranovic S \& Harris MP 2016 Parallelism and epistasis in skeletal evolution identified through use of phylogenomic mapping strategies. Molecular Biology and Evolution 33 162-173. (https://doi.org/10.1093/molbev/msv208)

de Rooij DG 2001 Proliferation and differentiation of spermatogonial stem cells. Reproduction 121 347-354. (https://doi.org/10.1530/ rep.0.1210347)

Dranow DB, Tucker RP \& Draper BW 2013 Germ cells are required to maintain a stable sexual phenotype in adult zebrafish. Developmental Biology 376 43-50. (https://doi.org/10.1016/j.ydbio.2013.01.016)

Dranow DB, Hu K, Bird AM, Lawry ST, Adams MT, Sanchez A, Amatruda JF \& Draper BW 2016 Bmp15 is an oocyte-produced signal required for maintenance of the adult female sexual phenotype in zebrafish. PLoS Genetics 12 e1006323. (https://doi.org/10.1371/journal.pgen.1006323)

Feitsma H, Leal MC, Moens PB, Cuppen E \& Schulz RW 2007 Mlh1 deficiency in zebrafish results in male sterility and aneuploid as well as triploid progeny in females. Genetics 175 1561-1569. (https://doi. org/10.1534/genetics.106.068171)

Houwing S, Kamminga LM, Berezikov E, Cronembold D, Girard A, van den Elst H, Filippov DV, Blaser H, Raz E, Moens CB et al. 2007 A role for piwi and pirnas in germ cell maintenance and transposon silencing in zebrafish. Cell 129 69-82. (https://doi.org/10.1016/j.cell.2007.03.026)

Howe K, Clark MD, Torroja CF, Torrance J, Berthelot C, Muffato M, Collins JE, Humphray S, McLaren K, Matthews L et al. 2013 The zebrafish reference genome sequence and its relationship to the human genome. Nature 496 498-503. (https://doi.org/10.1038/nature12111)

Hsu C, Pan Y-J, Wang Y-W, Tong S-K \& Chung B 2018 Changes in the morphology and gene expression of developing zebrafish gonads. General and Comparative Endocrinology 265 154-159. (https://doi. org/10.1016/j.ygcen.2018.01.026)

Hu Y-C, de Rooij DG \& Page DC 2013 Tumor suppressor gene Rb is required for self-renewal of spermatogonial stem cells in mice. PNAS 110 12685-12690. (https://doi.org/10.1073/pnas.1311548110)

Kumar S, Stecher G \& Tamura K 2016 MEGA7: molecular evolutionary genetics analysis version 7.0 for bigger datasets. Molecular Biology and Evolution 33 1870-1874. (https://doi.org/10.1093/molbev/msw054)

Laurenti E, Frelin C, Xie S, Ferrari R, Dunant CF, Zandi S, Neumann A, Plumb I, Doulatov S, Chen J et al. 2015 CDK6 levels regulate quiescence exit in human hematopoietic stem cells. Cell Stem Cell 16 302-313. (https://doi.org/10.1016/j.stem.2015.01.017)

Leal MC, Cardoso ER, Nóbrega RH, Batlouni SR, Bogerd J, França LR \& Schulz RW 2009 Histological and stereological evaluation of zebrafish (Danio rerio) spermatogenesis with an emphasis on spermatogonial generations. Biology of Reproduction 81 177-187. (https://doi. org/10.1095/biolreprod.109.076299)

Lubzens E, Young G, Bobe J \& Cerdà J 2010 Oogenesis in teleosts: how eggs are formed. General and Comparative Endocrinology 165 367-389. (https://doi.org/10.1016/j.ygcen.2009.05.022)

Luzio A, Coimbra AM, Benito C, Fontaínhas-Fernandes AA \& Matos M 2015 Screening and identification of potential sex-associated sequences 
in Danio rerio. Molecular Reproduction and Development 82 756-764. (https://doi.org/10.1002/mrd.22508)

Maack G \& Segner H 2003 Morphological development of the gonads in zebrafish. Journal of Fish Biology 62 895-906. (https://doi.org/10.1046/ j.1095-8649.2003.00074.x)

Malumbres M 2014 Cyclin-dependent kinases. Genome Biology 15122. (https://doi.org/10.1186/gb4184)

Malumbres M, Sotillo R, Santamaría D, Galán J, Cerezo A, Ortega S, Dubus P \& Barbacid M 2004 Mammalian cells cycle without the D-type cyclin-dependent kinases Cdk4 and Cdk6. Cell 118 493-504. (https:// doi.org/10.1016/j.cell.2004.08.002)

Morelli MA \& Cohen PE 2005 Not all germ cells are created equal: aspects of sexual dimorphism in mammalian meiosis. Reproduction 130 761-781. (https://doi.org/10.1530/rep.1.00865)

Moreno-Mateos MA, Vejnar CE, Beaudoin J-D, Fernandez JP, Mis EK, Khokha MK \& Giraldez AJ 2015 CRISPRscan: designing highly efficient sgRNAs for CRISPR-Cas9 targeting in vivo. Nature Methods 12 982-988. (https://doi.org/10.1038/nmeth.3543)

Nóbrega RH, Greebe CD, van de Kant H, Bogerd J, de França LR \& Schulz RW 2010 Spermatogonial stem cell niche and spermatogonial stem cell transplantation in zebrafish. PLOS ONE 5 1-16. (https://doi. org/10.1371/journal.pone.0012808)

Rane SG, Dubus P, Mettus RV, Galbreath EJ, Boden G, Reddy EP \& Barbacid M 1999 Loss of Cdk4 expression causes insulin-deficient diabetes and Cdk4 activation results in beta-islet cell hyperplasia. Nature Genetics 22 44-52. (https://doi.org/10.1038/8751)

Risal S, Adhikari D \& Liu K 2016 Animal models for studying the in vivo functions of cell cycle CDKs. Methods in Molecular Biology 1336 155-166. (https://doi.org/10.1007/978-1-4939-2926-9_13)

Rodríguez-Marí A, Cañestro C, Bremiller RA, Nguyen-Johnson A Asakawa K, Kawakami K \& Postlethwait JH 2010 Sex reversal in zebrafish fancl mutants is caused by Tp53-mediated germ cell apoptosis. PLoS Genetics 6 e1001034. (https://doi.org/10.1371/journal.pgen.1001034)

Rodríguez-Marí A, Wilson C, Titus TA, Cañestro C, BreMiller RA, Yan YL, Nanda I, Johnston A, Kanki JP, Gray EM et al. 2011 Roles of brca2 (fancd1) in oocyte nuclear architecture, gametogenesis, gonad tumors, and genome stability in zebrafish. PLoS Genetics 7 e1001357. (https:// doi.org/10.1371/journal.pgen.1001357)

Saito K, Siegfried KR, Nüsslein-Volhard C \& Sakai N 2011 Isolation and cytogenetic characterization of zebrafish meiotic prophase I mutants. Developmental Dynamics 240 1779-1792. (https://doi.org/10.1002/ dvdy.22661)

Saito K, Sakai C, Kawasaki T \& Sakai N 2014 Telomere distribution pattern and synapsis initiation during spermatogenesis in zebrafish. Developmental Dynamics 243 1448-1456. (https://doi.org/10.1002/ dvdy.24166)
Schulz RW, de França LR, Lareyre J-J, LeGac F, Chiarini-Garcia H, Nobrega RH, Miura T 2010 Spermatogenesis in fish. General and Comparative Endocrinology 165 390-411. (https://doi.org/10.1016/j. ygcen.2009.02.013)

Sherr CJ \& Roberts JM 2004 Living with or without cyclins and cyclindependent kinases. Genes and Development 18 2699-2711. (https:// doi.org/10.1101/gad.1256504)

Siegfried KR \& Nüsslein-Volhard C 2008 Germ line control of female sex determination in zebrafish. Developmental Biology 324 277-287. (https://doi.org/10.1016/j.ydbio.2008.09.025)

Slanchev K, Stebler J, de la Cueva-Méndez G \& Raz E 2005 Development without germ cells: the role of the germ line in zebrafish sex differentiation. PNAS 102 4074-4079. (https://doi.org/10.1073/pnas.0407475102)

Smith A, Zhang J, Guay D, Quint E, Johnson A \& Akimenko MA 2008 Gene expression analysis on sections of zebrafish regenerating fins reveals limitations in the whole-mount in situ hybridization method. Developmental Dynamics 237 417-425. (https://doi.org/10.1002/dvdy.21417)

Tsutsui T, Hesabi B, Moons DS, Pandolfi PP, Hansel KS, Koff A \& Kiyokawa H 1999 Targeted disruption of CDK4 delays cell cycle entry with enhanced p27(Kip1) activity. Molecular and Cellular Biology 19 7011-7019. (https://doi.org/10.1128/MCB.19.10.7011)

Tzung K-W, Goto R, Saju JM, Sreenivasan R, Saito T, Arai K, Yamaha E, Hossain MS, Calvert MK \& Orbán L 2015 Early depletion of primordial germ cells in zebrafish promotes testis formation. Stem Cell Reports $\mathbf{4}$ 61-73. (https://doi.org/10.1016/j.stemcr.2014.10.011)

Wilson CA, High SK, McCluskey BM, Amores A, Yan Y-I, Titus TA, Anderson JL, Batzel P, Carvan MJ, Schartl M et al. 2014 Wild sex in Zebrafish: loss of the natural sex determinant in domesticated strains. Genetics 198 1291-1308. (https://doi.org/10.1534/genetics.114.169284)

Wolgemuth DJ, Manterola M \& Vasileva A 2013 Role of cyclins in controlling progression of mammalian spermatogenesis. International Journal of Developmental Biology 57 159-168. (https://doi.org/10.1387/ ijdb.130047av)

Yang Q-E, Gwost I, Oatley MJ \& Oatley JM 2013 Retinoblastoma protein (RB1) controls fate determination in stem cells and progenitors of the mouse male germline. Biology of Reproduction 89 113. (https://doi. org/10.1095/biolreprod.113.113159)

Received 25 July 2018

First decision 12 September 2018

Revised manuscript received 28 January 2019

Accepted 14 February 2019 